\title{
A GENERALIZED TELEGRAPH PROCESS WITH VELOCITY DRIVEN BY RANDOM TRIALS
}

\author{
IRENE CRIMALDI,* IMT \\ ANTONIO DI CRESCENZO, ${ }^{* * * * *}$ Università di Salerno \\ ANTONELLA IULIANO, **** Istituto per le Applicazioni del Calcolo 'Mauro Picone' \\ BARBARA MARTINUCCI, ${ }^{* * * * * * *}$ Università di Salerno
}

\begin{abstract}
We consider a random trial-based telegraph process, which describes a motion on the real line with two constant velocities along opposite directions. At each epoch of the underlying counting process the new velocity is determined by the outcome of a random trial. Two schemes are taken into account: Bernoulli trials and classical Pólya urn trials. We investigate the probability law of the process and the mean of the velocity of the moving particle. We finally discuss two cases of interest: (i) the case of Bernoulli trials and intertimes having exponential distributions with linear rates (in which, interestingly, the process exhibits a logistic stationary density with nonzero mean), and (ii) the case of Pólya trials and intertimes having first gamma and then exponential distributions with constant rates.
\end{abstract}

Keywords: Telegraph process; random intertime; random velocity; Bernoulli scheme; Pólya urn model; logistic stationary density

2010 Mathematics Subject Classification: Primary 60K15

Secondary $60 \mathrm{~K} 37$

\section{Introduction}

Since the 1950s several authors have investigated the (integrated) telegraph process as a realistic model of random motion (see [4], [20], and [23]). Such a process describes the motion of a particle on the real line characterized by a constant speed, the direction being reversed at the random epochs of a Poisson process. The probability density of the particle's position satisfies a hyperbolic differential equation, whose probabilistic properties have been studied by, for instance, Orsingher [26], [27], Foong and Kanno [19], and, more recently, Beghin et al. [5]. See also the book of Pinsky [28], in which many results on the telegraph process and its generalizations were given, and many applications were discussed. Certain one-dimensional generalizations of the telegraph process focus on cases in which the intertimes between two consecutive changes of direction are characterized by a variety of distributions. We recall the cases of the Erlang distribution (see [14]), the gamma distribution (see [15]), and the exponential

Received 4 September 2012; revision received 11 February 2013.

* Postal address: Institute for Advanced Studies, IMT, Piazza San Ponziano 6, I-55100 Lucca, Italy.

Email address: irene.crimaldi@imtlucca.it

** Postal address: Dipartimento di Matematica, Università di Salerno, Via Ponte don Melillo, Fisciano (SA), I-84084, Italy.

*** Email address: adicrescenzo@unisa.it

**** Postal address: Istituto per le Applicazioni del Calcolo 'Mauro Picone', CNR, Via Pietro Castellino 111, I-80131

Napoli, Italy. Email address: a.iuliano@na.iac.cnr.it

***** Email address: bmartinucci@unisa.it 
distribution with linearly increasing rate (see [17]). Moreover, a generalized telegraph process governed by an alternating renewal process was studied by Zacks [33], whereas Iacus [22] gave a rare example where an explicit probability law is obtained for an inhomogeneous telegraph process.

In this paper we aim to study the telegraph model subject to a further source of randomness, by assuming that the velocity of the moving particle is driven by random trials. Specifically, we deal with a two-velocity random motion on the real line where, differently from the classical telegraph process whose positive and negative velocities are alternating, at each time epoch the new velocity is determined by the outcome of a random trial. The latter follows one out of two schemes: the Bernoulli scheme, which acts with independence, and the classical Pólya scheme (cf. [24] and [29]), where the outcome of each trial depends on the outcomes of the previous trials. We note that the inclusion of random trials in finite-velocity random evolutions allows us to describe some real situations of interest, such as the motions of particles subject to collisions, whose effects may produce direction changes.

Some novelties with respect to various finite-velocity random motions are mentioned in Section 2, with special reference to models with random velocities. We recall that investigations on the telegraph process with random velocities have been performed recently by Stadje and Zacks [31] and De Gregorio [11]. Moreover, some recent contributions on multidimensional motions characterized by a finite speed and randomly distributed directions are given in De Gregorio [12] and De Gregorio and Orsingher [13].

The paper is organized as follows. In Section 2 we describe in detail the mathematical model of the motion. In Section 3 we investigate the probability law and the conditional mean velocity of the process. Then, in Section 4, for the Bernoulli scheme, we discuss the case in which the random intertimes between consecutive trials are exponentially distributed with linearly increasing rates. In this case we obtain the probability density of the process in closed form, we show that it possesses a logistic stationary density, and then we express the conditional mean velocity of the process in terms of hypergeometric functions. Finally, in Section 5, for the Pólya scheme, we discuss the case in which the first random intertimes in both directions are gamma distributed, whereas all remaining intertimes are exponentially distributed. We obtain the probability density in closed form and the conditional mean velocity as a series of Gauss hypergeometric functions. For the reader's convenience, the paper is enriched by two appendices containing definitions and formulae used in the proofs.

\section{Stochastic model}

Let $\left\{\left(S_{t}, V_{t}\right) ; t \geq 0\right\}$ be a continuous-time stochastic process, where $S_{t}$ and $V_{t}$ respectively denote the position and velocity at time $t$ of the moving particle. The motion is characterized by two velocities, $c$ and $-v$ with $c, v>0$, and its direction is specified by the sign of the velocity. At time $T_{0}=0$ the particle starts from the origin; thus, $S_{0}=0$. The initial velocity $V_{0}$ is determined by the outcome $X_{1}$ of the first random trial. At the random time $T_{1}>0$ the particle is subject to an event, whose effect potentially changes the velocity according to the outcome $X_{2}$ of the second random trial. This behavior is repeated cyclically at every instant of a sequence of random epochs $T_{0}=0<T_{1}<T_{2}<T_{3}<\cdots$. We assume that the time interval durations $\left[T_{n}, T_{n+1}\right), n=0,1,2, \ldots$, constitute a sequence of nonnegative random variables. More precisely, let $U_{k}$ and $D_{k}$ denote the random durations of the $k$ th time period during which the particle moves forward with velocity $c$ and, respectively, backward with velocity $-v$. Furthermore, $\left\{U_{k} ; k \geq 1\right\}$ and $\left\{D_{k} ; k \geq 1\right\}$ are mutually independent sequences of nonnegative and absolutely continuous independent random variables. Denoting by $Z_{n}$ the velocity of the 
particle during the interval $\left[T_{n}, T_{n+1}\right)$, we assume that $\left\{Z_{n} ; n \geq 0\right\}$ is a sequence of random variables governed by the sequence $\left\{X_{n} ; n \geq 1\right\}$ of random trial outcomes. Moreover, we assume that the collection $\left\{U_{k}, D_{k} ; n \geq 1\right\}$ is independent of $\left\{X_{n} ; n \geq 1\right\}$.

In this paper we focus on the case in which the random trials $\left\{X_{n} ; n \geq 1\right\}$ are the outcomes of a sequence of indicator functions such that

$$
\mathbb{P}\left\{Z_{0}=c\right\}=\mathbb{P}\left\{X_{1}=1\right\}=\frac{b}{b+r}, \quad \mathbb{P}\left\{Z_{0}=-v\right\}=\mathbb{P}\left\{X_{1}=0\right\}=\frac{r}{b+r},
$$

and, for $n \geq 1$,

$$
\begin{gathered}
\mathbb{P}\left\{Z_{n}=c \mid g_{n}\right\}=\mathbb{P}\left\{X_{n+1}=1 \mid g_{n}\right\}=\frac{b+A \sum_{k=1}^{n} X_{k}}{b+r+A n}, \\
\mathbb{P}\left\{Z_{n}=-v \mid g_{n}\right\}=\mathbb{P}\left\{X_{n+1}=0 \mid g_{n}\right\}=\frac{r+A \sum_{k=1}^{n}\left(1-X_{k}\right)}{b+r+A n},
\end{gathered}
$$

where $b$ and $r$ are positive constants, $A$ is a nonnegative constant, $g_{0}=\{\varnothing, \Omega\}$, and $g_{n}=$ $\sigma\left(X_{1}, \ldots, X_{n}\right)$ for $n \geq 1$.

We can distinguish two cases.

Case (i): $A=0$. In this case the random trials $\left\{X_{n} ; n \geq 1\right\}$ are independent, i.e. they are a Bernoulli scheme with parameter

$$
p=\frac{b}{b+r} .
$$

Case (ii): $A>0$. In this case $\left\{X_{n} ; n \geq 1\right\}$ is a sequence of outcomes of drawing balls from an urn that initially contains $b$ black balls and $r$ red balls, and that is updated according to the classical Pólya urn scheme: the drawn ball is returned to the urn together with $A>0$ balls of the same color. (We recall that, from a mathematical point of view, in this case the parameters $b, r$, and $A$ of the model can be real, not necessarily integer.) The outcome $X_{1}=1$ means that the drawn ball is black; otherwise, we have $X_{1}=0$. In this case the random trials $\left\{X_{n} ; n \geq 1\right\}$ are not independent, but only exchangeable (see [2]). We recall that urn schemes are used in many applications in order to model the so-called preferential attachment principle, which is a key feature governing the dynamics of many economic, social, and biological systems. It can be formulated as follows: the greater the number of times we observe a certain event, the higher the probability of occurrence of that event the next time.

In Section 5.1 we discuss an extension of the above setting to the case in which $\left\{X_{n} ; n \geq 1\right\}$ is the sequence of outcomes of drawings from a randomly reinforced urn.

We note that Stadje and Zacks [31] studied a telegraph process with random velocities, where at each epoch of a homogeneous Poisson process the new velocity of the motion is chosen according to a common density, independently of the previous velocities and of the Poisson process. Hence, in particular, the velocities are a sequence of independent and identically distributed (i.i.d.) random variables and, in Section 5 of [31], the first-exit time of the process through a positive constant is investigated in the special case of two-valued random velocities. This schema corresponds to our case (i), where at each random epoch a Bernoulli trial occurs. However, there is a significant difference: whereas in [31] the random durations are independent of the random velocities, our model is based on more general assumptions, which involve nonidentically distributed durations of random intervals $\left[T_{n}, T_{n+1}\right)$, depending on the values 

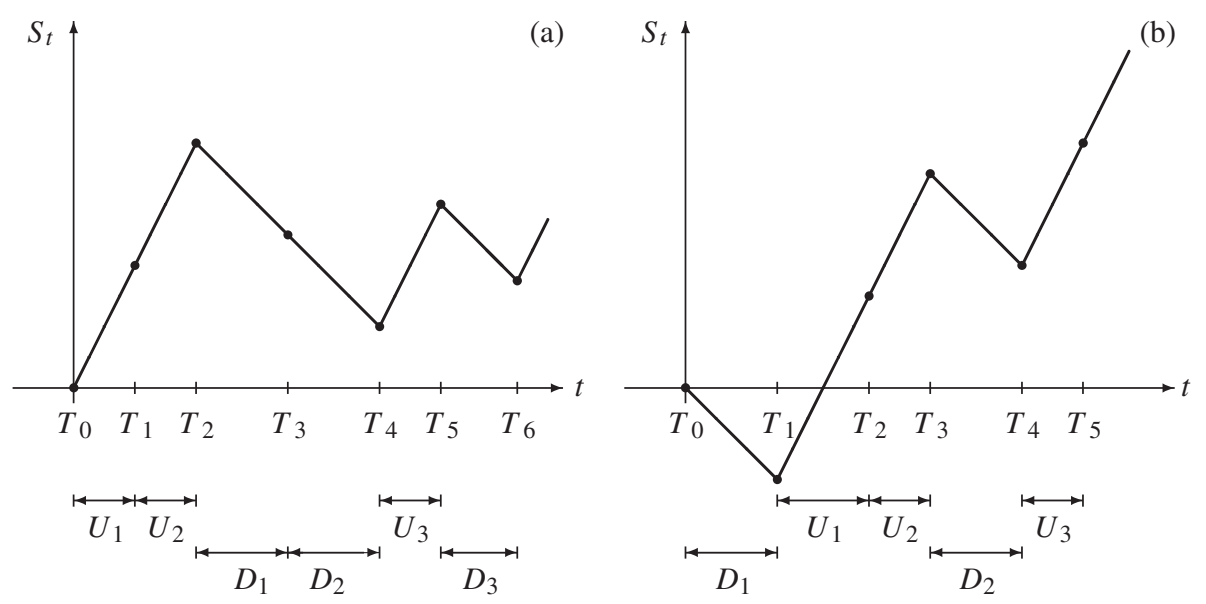

FIGURE 1: Sample paths of $S_{t}$ with (a) $V_{0}=c$ and (b) $V_{0}=-v$.

taken by the velocities. Moreover, our model includes the case of nonindependent random velocities (case (ii)). These two facts are also novelties with respect to [11], where, as in [31], a homogeneous Poisson process governs the velocity changes that form a sequence of i.i.d. random variables, independent of the Poisson process.

Let $M_{t}$ be the stochastic process which counts the number of epochs $T_{i}$ (with $i \geq 1$ ) occurring before $t$, i.e.

$$
M_{t}=\max \left\{i \geq 1: T_{i} \leq t\right\}, \quad t>0 .
$$

It is worth noting that the position and velocity of the particle at time $t$ can thus be formally expressed as

$$
V_{t}=Z_{M_{t}}, \quad S_{t}=\int_{0}^{t} V_{s} \mathrm{~d} s, \quad t>0 .
$$

In Figure 1 we present two sample path examples of $S_{t}$, with indications of the random intertimes $U_{k}$ and $D_{k}$, where the sequence $\left\{X_{n} ; n \geq 1\right\}$ takes values $\{1,1,0,0,1,0,1, \ldots\}$ (left-hand plot) and $\{0,1,1,0,1,1, \ldots\}$ (right-hand plot).

The following stochastic equation holds:

$$
S_{T_{k+1}}=S_{T_{k}}+W_{k}, \quad k \geq 0
$$

Here $\left\{W_{k} ; k \geq 0\right\}$ is the sequence of random variables defined by

$$
W_{0}= \begin{cases}c U_{1} & \text { if } Z_{0}=c \\ -v D_{1} & \text { if } Z_{0}=-v\end{cases}
$$

and, for $k \geq 1$ and $1 \leq j \leq k+1$,

$$
W_{k}= \begin{cases}c U_{j} & \text { if } Z_{k}=c \text { and } X_{1}+N_{k-1}=j-1, \\ -v D_{j} & \text { if } Z_{k}=-v \text { and } X_{1}+N_{k-1}=k-j+1,\end{cases}
$$

where $N_{k-1}$ is the random variable that counts the number of random trials yielding velocity $c$ among the trials going from the second to the $k$ th trial, i.e.

$$
N_{k-1}=\sum_{i=1}^{k-1} \mathbf{1}_{\left\{Z_{i}=c\right\}}=\sum_{i=1}^{k-1} X_{i+1}=\sum_{h=2}^{k} X_{h}, \quad k \geq 2 .
$$


For convenience, we also set $N_{0}=0$. In Appendix A we give some useful formulae for the probability distribution of $N_{k-1}$ conditioned on the initial velocity for the two different schemes.

Throughout the paper, we denote by $f_{U_{k}}, F_{U_{k}}$, and $\bar{F}_{U_{k}}$ the probability densities, cumulative distribution functions, and tail distribution functions of $U_{k}$, respectively, and, similarly, $f_{D_{k}}$, $F_{D_{k}}$, and $\bar{F}_{D_{k}}$ the probability densities, cumulative distribution functions, and tail distribution functions of $D_{k}$, respectively. Moreover, $f_{U}^{(k)}, f_{D}^{(k)}, F_{U}^{(k)}, F_{D}^{(k)}$, and $\bar{F}_{U}^{(k)}, \bar{F}_{D}^{(k)}$ will respectively denote the probability densities, cumulative distribution functions, and tail distribution functions of the partial sums

$$
U^{(k)}=U_{1}+U_{2}+\cdots+U_{k}, \quad D^{(k)}=D_{1}+D_{2}+\cdots+D_{k}, \quad k \geq 1 .
$$

\section{Probability law and mean velocity}

At time $t=0$ we assume that $S_{0}=0$, so that at time $t>0$ the particle is located in the domain $[-v t, c t]$. The probability law of $S_{t}, t>0$, thus possesses a discrete component on the points $-v t$ and $c t$, and an absolutely continuous component over $(-v t, c t)$, which will be investigated in the sequel.

Proposition 1. For all $t>0$, we have

$$
\mathbb{P}\left\{S_{t}=c t\right\}=\frac{b}{b+r} \bar{F}_{U_{1}}(t)+\sum_{k=1}^{+\infty} \mathbb{P}\left\{Z_{0}=c, N_{k}=k\right\}\left[F_{U}^{(k)}(t)-F_{U}^{(k+1)}(t)\right],
$$

where

$$
\mathbb{P}\left\{Z_{0}=c, N_{k}=k\right\}= \begin{cases}p^{k+1} & \text { if } A=0, \\ \frac{b}{b+r}\left(\frac{b+A}{A}\right)_{k}\left[\left(\frac{b+A+r}{A}\right)_{k}\right]^{-1} & \text { if } A>0 .\end{cases}
$$

Similarly, we have

$$
\mathbb{P}\left\{S_{t}=-v t\right\}=\frac{r}{b+r} \bar{F}_{D_{1}}(t)+\sum_{k=1}^{+\infty} \mathbb{P}\left\{Z_{0}=-v, N_{k}=0\right\}\left[F_{D}^{(k)}(t)-F_{D}^{(k+1)}(t)\right],
$$

where

$$
\mathbb{P}\left\{Z_{0}=-v, N_{k}=0\right\}= \begin{cases}(1-p)^{k+1} & \text { if } A=0, \\ \frac{r}{b+r}\left(\frac{r+A}{A}\right)_{k}\left[\left(\frac{b+A+r}{A}\right)_{k}\right]^{-1} & \text { if } A>0 .\end{cases}
$$

Proof. First, note that, for $y \in\{-v, c\}$, the condition $S_{t}=y t$ implies that $V_{0}=y$ and $V_{t}=y$ almost surely. Conditioning on $M_{t}=k$ and recalling (6), we have

$$
\begin{aligned}
\mathbb{P}\left\{S_{t}=c t\right\} & =\mathbb{P}\left\{S_{t}=c t, V_{t}=c, V_{0}=c\right\} \\
& =\sum_{k=0}^{+\infty} \mathbb{P}\left\{Z_{0}=c, N_{k}=k\right\} \mathbb{P}\left\{M_{t}=k \mid Z_{0}=c, N_{k}=k\right\} .
\end{aligned}
$$

Equation (7) thus easily follows from (1) and from (45) and (50) below for $j=k-1$. In the same way, (8) follows from (1) and from (48) and (53) below. 
Let us now define the probability density of $S_{t}, t>0$, conditional on the initial velocity $y \in\{-v, c\}$ :

$$
p(x, t \mid y)=\frac{\partial}{\partial x} \mathbb{P}\left\{S_{t} \leq x \mid V_{0}=y\right\}, \quad x \in(-v t, c t) .
$$

We remark that, for $t>0$,

$$
\mathbb{P}\left\{S_{t}=y t \mid V_{0}=y\right\}+\int_{-v t}^{c t} p(x, t \mid y) \mathrm{d} x=1, \quad y \in\{-v, c\} .
$$

The density of the particle position is

$$
p(x, t)=\frac{\partial}{\partial x} \mathbb{P}\left\{S_{t} \leq x\right\}=\frac{b}{b+r} p(x, t \mid c)+\frac{r}{b+r} p(x, t \mid-v),
$$

where $p(x, t \mid y)$, defined in (9), can be expressed as

$$
p(x, t \mid y)=f(x, t \mid y)+b(x, t \mid y) .
$$

Here $f$ and $b$ denote the densities of the particle's position when the motion at time $t$ is characterized by forward and backward velocities, respectively, i.e.

$$
\begin{aligned}
f(x, t \mid y) & =\frac{\partial}{\partial x} \mathbb{P}\left\{S_{t} \leq x, V_{t}=c \mid V_{0}=y\right\}, \\
b(x, t \mid y) & =\frac{\partial}{\partial x} \mathbb{P}\left\{S_{t} \leq x, V_{t}=-v \mid V_{0}=y\right\},
\end{aligned}
$$

for $x$ in $(-v t, c t)$. Then, for $y \in\{-v, c\}$, we can write

$$
f(x, t \mid y)=\sum_{k=1}^{+\infty} f_{k}(x, t \mid y), \quad b(x, t \mid y)=\sum_{k=1}^{+\infty} b_{k}(x, t \mid y),
$$

where, for $x$ in $(-v t, c t)$,

$$
\begin{aligned}
& f_{k}(x, t \mid y)=\frac{\partial}{\partial x} \mathbb{P}\left\{S_{t} \leq x, V_{t}=c, M_{t}=k \mid V_{0}=y\right\}, \\
& b_{k}(x, t \mid y)=\frac{\partial}{\partial x} \mathbb{P}\left\{S_{t} \leq x, V_{t}=-v, M_{t}=k \mid V_{0}=y\right\},
\end{aligned}
$$

with $M_{t}$ defined in (4). It is worth recalling that, in the case of a telegraph process driven by i.i.d. random velocities and an independent homogeneous Poisson process, a two-dimensional renewal equation for $p(x, t)$ is given in [31, Equation (2.5)]. However, as pointed out by the authors, it is quite difficult to solve analytically. In our case, hereafter we develop a different approach, based on suitable conditioning. We first give expressions for the densities $f_{k}$ and $b_{k}$ conditioned by $V_{0}=c$. Note that $f_{1}(x, t \mid c)=0$.

Theorem 1. For $t>0$ and $-v t<x<c t$, densities (13) and (14) can be expressed as

$$
\begin{aligned}
f_{k}(x, t \mid c)= & \frac{1}{c+v} \sum_{j=0}^{k-2} \mathbb{P}\left\{N_{k-1}=j, Z_{k}=c \mid Z_{0}=c\right\} \\
& \times f_{D}^{(k-j-1)}\left(t-\tau_{*}\right) \int_{t-\tau_{*}}^{t} f_{U}^{(j+1)}\left(s-t+\tau_{*}\right) \bar{F}_{U_{j+2}}(t-s) \mathrm{d} s, \quad k \geq 2,
\end{aligned}
$$


and

$$
\begin{gathered}
b_{k}(x, t \mid c) \\
=\frac{1}{c+v}\left\{\mathbb{P}\left\{N_{k-1}=k-1, Z_{k}=-v \mid Z_{0}=c\right\} f_{U}^{(k)}\left(\tau_{*}\right) \bar{F}_{D_{1}}\left(t-\tau_{*}\right)\right. \\
+\mathbf{1}_{\{k \geq 2\}} \sum_{j=0}^{k-2} \mathbb{P}\left\{N_{k-1}=j, Z_{k}=-v \mid Z_{0}=c\right\} f_{U}^{(j+1)}\left(\tau_{*}\right) \\
\left.\quad \times \int_{\tau_{*}}^{t} f_{D}^{(k-j-1)}\left(s-\tau_{*}\right) \bar{F}_{D_{k-j}}(t-s) \mathrm{d} s\right\}, \quad k \geq 1,
\end{gathered}
$$

where

$$
\tau_{*}=\tau_{*}(x, t)=\frac{v t+x}{c+v},
$$

and where $\mathbb{P}\left\{N_{k-1}=j, Z_{k}=c \mid Z_{0}=c\right\}$ and $\mathbb{P}\left\{N_{k-1}=j, Z_{k}=-v \mid Z_{0}=c\right\}$ are given respectively by (45) and (46) below if $A=0$, and by (50) and (51) below if $A>0$.

Proof. Recalling (13), for $t>0,-v t<x<c t$, and $k \geq 2$, we have

$$
\begin{gathered}
f_{k}(x, t \mid c) \mathrm{d} x=\int_{0}^{t} \mathbb{P}\left\{T_{k} \in \mathrm{d} s, Z_{k}=c, S_{s}+c(t-s) \in \mathrm{d} x, T_{k+1}-T_{k}>t-s,\right. \\
\left.0 \leq N_{k-1} \leq k-2 \mid Z_{0}=c\right\} .
\end{gathered}
$$

(The $k=1$ case does not give an absolutely continuous component.) Conditioning on $N_{k-1}$, and taking into account the number of time periods during which the particle moved forward and backward, we obtain

$$
\begin{aligned}
& f_{k}(x, t \mid c) \mathrm{d} x \\
& =\sum_{j=0}^{k-2} \int_{0}^{t} \mathbb{P}\left\{U^{(j+1)}+D^{(k-j-1)} \in \mathrm{d} s, c U^{(j+1)}-v D^{(k-j-1)}+c(t-s) \in \mathrm{d} x\right\} \\
& \quad \times \mathbb{P}\left\{U_{j+2}>t-s\right\} \mathbb{P}\left\{N_{k-1}=j, Z_{k}=c \mid Z_{0}=c\right\} .
\end{aligned}
$$

Note that the conditions $S_{s}+c(t-s)=x$ and $S_{s} \geq-v s$ yield $s \geq(c t-x) /(c+v)=t-\tau_{*}$. This inequality and the independence of $U^{(k)}$ and $\bar{D}^{(k)}$ thus give

$$
\begin{array}{rl}
f_{k}(x, t \mid c)=\sum_{j=0}^{k-2} & \mathbb{P}\left\{N_{k-1}=j, Z_{k}=c \mid Z_{0}=c\right\} \\
& \times \int_{t-\tau_{*}}^{t} h(s, x-c(t-s)) \mathbb{P}\left\{U_{j+2}>t-s\right\} \mathrm{d} s,
\end{array}
$$

where $h(\cdot, \cdot)$ is the joint probability density of $\left(U^{(j+1)}+D^{(k-j-1)}, c U^{(j+1)}-v D^{(k-j-1)}\right)$. Since

$$
h(s, x-c(t-s))=\frac{1}{c+v} f_{U}^{(j+1)}\left(s-\frac{c t-x}{c+v}\right) f_{D}^{(k-j-1)}\left(\frac{c t-x}{c+v}\right),
$$

(15) now follows by recalling (17). Equation (16) can be obtained in a similar way. Indeed, for $k \geq 1$,

$$
\begin{gathered}
b_{k}(x, t \mid c) \mathrm{d} x=\int_{0}^{t} \mathbb{P}\left\{T_{k} \in \mathrm{d} s, Z_{k}=-v, S_{s}-v(t-s) \in \mathrm{d} x, T_{k+1}-T_{k}>t-s,\right. \\
\left.0 \leq N_{k-1} \leq k-1 \mid Z_{0}=c\right\} .
\end{gathered}
$$


Conditioning on $N_{k-1}$, and taking into account the number of time periods during which the particle moved forward and backward, we obtain, for $k \geq 1$,

$$
\begin{aligned}
& b_{k}(x, t \mid c) \mathrm{d} x \\
& =\int_{0}^{t} \mathbb{P}\left\{U^{(k)} \in \mathrm{d} s, c U^{(k)}-v(t-s) \in \mathrm{d} x\right\} \\
& \quad \times \mathbb{P}\left\{D_{1}>t-s\right\} \mathbb{P}\left\{N_{k-1}=k-1, Z_{k}=-v \mid Z_{0}=c\right\} \\
& \quad+\sum_{j=0}^{k-2} \int_{0}^{t} \mathbb{P}\left\{U^{(j+1)}+D^{(k-j-1)} \in \mathrm{d} s, c U^{(j+1)}-v D^{(k-j-1)}-v(t-s) \in \mathrm{d} x\right\} \\
& \quad \times \mathbb{P}\left\{D_{k-j}>t-s\right\} \mathbb{P}\left\{N_{k-1}=j, Z_{k}=-v \mid Z_{0}=c\right\} .
\end{aligned}
$$

(Note that, for $k=1$, the term $\mathbb{P}\left\{N_{k-1}=k-1, Z_{k}=-v \mid Z_{0}=c\right\}$ reduces to $\mathbb{P}\left\{Z_{1}=\right.$ $\left.-v \mid Z_{0}=c\right\}$ and the sum on $j$ is equal to 0 .) For the first integral, the conditions $U^{(k)}=s$ and $c U^{(k)}-v(t-s)=x$ imply that $s=\tau_{*}$; for the second integral, the conditions $S_{s}-v(t-s)=x$ and $S_{s} \leq c s$ yield $s \geq(x+v t) /(c+v)=\tau_{*}$. This inequality and the independence of $U^{(k)}$ and $D^{(k)}$ thus give, for the above sum on $j$,

$$
\sum_{j=0}^{k-2} \mathbb{P}\left\{N_{k-1}=j, Z_{k}=-v \mid Z_{0}=c\right\} \int_{\tau_{*}}^{t} h(s, x+v(t-s)) \mathbb{P}\left\{D_{k-j}>t-s\right\} \mathrm{d} s,
$$

where, as above, $h(\cdot, \cdot)$ is the joint probability density of $\left(U^{(j+1)}+D^{(k-j-1)}, c U^{(j+1)}-\right.$ $\left.v D^{(k-j-1)}\right)$. Since

$$
h(s, x+v(t-s))=\frac{1}{c+v} f_{U}^{(j+1)}\left(\tau_{*}\right) f_{D}^{(k-j-1)}\left(s-\tau_{*}\right),
$$

we obtain (16).

Remark 1. Similarly to (15) and (16), when the initial velocity is negative, for all $t>0$ and $-v t<x<c t$, densities (13) and (14) are expressed as

$$
\begin{aligned}
& f_{k}(x, t \mid-v) \\
& =\frac{1}{c+v}\left\{\mathbb{P}\left\{N_{k-1}=0, Z_{k}=c \mid Z_{0}=-v\right\} f_{D}^{(k)}\left(t-\tau_{*}\right) \bar{F}_{U_{1}}\left(\tau_{*}\right)\right. \\
& +\mathbf{1}_{\{k \geq 2\}} \sum_{j=0}^{k-2} \mathbb{P}\left\{N_{k-1}=k-1-j, Z_{k}=c \mid Z_{0}=-v\right\} f_{D}^{(j+1)}\left(t-\tau_{*}\right) \\
& \left.\times \int_{t-\tau_{*}}^{t} f_{U}^{(k-j-1)}\left(s-t+\tau_{*}\right) \bar{F}_{U_{k-j}}(t-s) \mathrm{d} s\right\}, \quad k \geq 1, \\
& b_{k}(x, t \mid-v)=\frac{1}{c+v} \sum_{j=0}^{k-2} \mathbb{P}\left\{N_{k-1}=k-1-j, Z_{k}=-v \mid Z_{0}=-v\right\} f_{U}^{(k-j-1)}\left(\tau_{*}\right) \\
& \times \int_{\tau_{*}}^{t} f_{D}^{(j+1)}\left(s-\tau_{*}\right) \bar{F}_{D_{j+2}}(t-s) \mathrm{d} s, \quad k \geq 2,
\end{aligned}
$$

where $\tau_{*}$ is defined in (17), and where $\mathbb{P}\left\{N_{k-1}=k-1-j, Z_{k}=c \mid Z_{0}=-v\right\}$ and $\mathbb{P}\left\{N_{k-1}=k-1-j, Z_{k}=-v \mid Z_{0}=-v\right\}$ are respectively given by (47) and (48) below if $A=0$, and by (52) and (53) below if $A>0$. 
In the following proposition, conditional on a positive initial velocity, the mean of the process $V_{t}$ is expressed in terms of the cumulative distribution function of $T_{k}$. (Obviously, we can obtain a similar expression conditioning on a negative initial velocity.) We note that, by conditioning on the value of $N_{k-1}$ and using the independence of $U^{(j+1)}$ and $D^{(k-j-1)}$, it follows that

$$
F_{T_{k} \mid Z_{0}}(t \mid c)=\sum_{j=0}^{k-1} \mathbb{P}\left\{N_{k-1}=j \mid Z_{0}=c\right\} \mathbb{P}\left\{U^{(j+1)}+D^{(k-j-1)} \leq t\right\},
$$

where

$$
\begin{aligned}
\mathbb{P}\left\{U^{(j+1)}+D^{(k-j-1)} \leq t\right\} & =\int_{0}^{t} F_{D}^{(k-j-1)}(t-s) f_{U}^{(j+1)}(s) \mathrm{d} s \\
& =\int_{0}^{t} F_{U}^{(j+1)}(t-s) f_{D}^{(k-j-1)}(s) \mathrm{d} s .
\end{aligned}
$$

Proposition 2. For all $t>0$, we have

$$
\mathbb{E}\left[V_{t} \mid V_{0}=c\right]=c \bar{F}_{U_{1}}(t)+c \pi_{A} \sum_{k=1}^{+\infty} \phi_{k}(t \mid c)+(-v)\left(1-\pi_{A}\right) \sum_{k=1}^{+\infty} \psi_{k}(t \mid c),
$$

where $\pi_{A}=(b+A) /(b+A+r)$ and

$$
\begin{aligned}
& \phi_{k}(t \mid c)=F_{T_{k} \mid Z_{0}}(t \mid c)-\int_{0}^{t} F_{T_{k} \mid Z_{0}}(t-s \mid c) f_{U_{k+1}}(s) \mathrm{d} s, \\
& \psi_{k}(t \mid c)=F_{T_{k} \mid Z_{0}}(t \mid c)-\int_{0}^{t} F_{T_{k} \mid Z_{0}}(t-s \mid c) f_{D_{k+1}}(s) \mathrm{d} s .
\end{aligned}
$$

Proof. We recall that in both cases (by independence in the Bernoulli scheme and by exchangeability in the Pólya scheme), we have, for each $n \geq 2$,

$$
\mathbb{P}\left\{X_{n}=1 \mid X_{1}=1\right\}=\pi_{A}, \quad \mathbb{P}\left\{X_{n}=0 \mid X_{1}=1\right\}=1-\pi_{A} .
$$

Hence, for every positive integer $k \geq 1$, recalling (4) and the first equation of (5), we have

$$
\mathbb{E}\left[V_{t} \mid V_{0}=c\right]=\mathbb{E}\left[Z_{M_{t}} \mid Z_{0}=c\right]=c \bar{F}_{U_{1}}(t)+\sum_{k=1}^{+\infty} \mathbb{E}\left[Z_{k} \mathbf{1}_{\left\{T_{k} \leq t<T_{k+1}\right\}} \mid Z_{0}=c\right] .
$$

Since, for $k \geq 1$,

$$
\begin{aligned}
& \mathbb{E}\left[Z_{k} \mathbf{1}_{\left\{T_{k} \leq t<T_{k+1}\right\}} \mid Z_{0}=c\right] \\
&=c \mathbb{P}\left\{Z_{k}=c \mid Z_{0}=c\right\} \mathbb{E}\left[\mathbf{1}_{\left\{T_{k} \leq t<T_{k+1}\right\}} \mid Z_{0}=c, Z_{k}=c\right] \\
&+(-v) \mathbb{P}\left\{Z_{k}=-v \mid Z_{0}=c\right\} \mathbb{E}\left[\mathbf{1}_{\left\{T_{k} \leq t<T_{k+1}\right\}} \mid Z_{0}=c, Z_{k}=-v\right] \\
&= c \mathbb{P}\left\{X_{k+1}=1 \mid X_{1}=1\right\} \mathbb{P}\left\{T_{k} \leq t<T_{k+1} \mid Z_{0}=c, Z_{k}=c\right\} \\
&+(-v) \mathbb{P}\left\{X_{k+1}=0 \mid X_{1}=1\right\} \mathbb{P}\left\{T_{k} \leq t<T_{k+1} \mid Z_{0}=c, Z_{k}=-v\right\} \\
&= c \pi_{A} \int_{0}^{\infty} \mathbb{P}\left\{t-s<T_{k} \leq t \mid Z_{0}=c\right\} f_{U_{k+1}}(s) \mathrm{d} s \\
&+(-v)\left(1-\pi_{A}\right) \int_{0}^{\infty} \mathbb{P}\left\{t-s<T_{k} \leq t \mid Z_{0}=c\right\} f_{D_{k+1}}(s) \mathrm{d} s \\
&= c \pi_{A} \phi_{k}(t \mid c)+(-v)\left(1-\pi_{A}\right) \psi_{k}(t \mid c) .
\end{aligned}
$$

Equation (20) now follows by using (21). 
The following remark turns out to be useful in some particular cases.

Remark 2. It is worth noting that, using (18) and (19), (21) can be rewritten as

$$
\begin{array}{rl}
\phi_{k}(t \mid c)=\sum_{j=0}^{k-1} & \mathbb{P}\left\{N_{k-1}=j \mid Z_{0}=c\right\} \\
\quad \times \int_{0}^{t} F_{D}^{(k-j-1)}(t-y)\left[f_{U}^{(j+1)}(y)-f_{U^{(j+1)}+U_{k+1}}(y)\right] \mathrm{d} y \\
\psi_{k}(t \mid c)=\sum_{j=0}^{k-1} & \mathbb{P}\left\{N_{k-1}=j \mid Z_{0}=c\right\} \\
& \times \int_{0}^{t} F_{U}^{(j+1)}(t-y)\left[f_{D}^{(k-j-1)}(y)-f_{D^{(k-j-1)}+D_{k+1}}(y)\right] \mathrm{d} y .
\end{array}
$$

The above quantities can be easily computed when the random variables $U_{k}$ (and $D_{k}$ ) are gamma distributed with the same scale parameter, since it is well known that, by independence, the sums of the involved random variables are still gamma distributed.

Remark 3. In the special case in which $\left(Z_{k}\right)_{k}$ and $\left(T_{k+1}-T_{k}\right)_{k}$ are independent, we have $U_{1}$ and $D_{1}$ identically distributed and

$$
\phi_{k}(t \mid c)=\psi_{k}(t \mid c)=\mathbb{P}\left\{T_{k} \leq t<T_{k+1}\right\},
$$

so we can write (20) as

$$
\mathbb{E}\left[V_{t} \mid V_{0}=c\right]=c \bar{F}_{T_{1}}(t)+\mathbb{E}\left[Z_{1} \mid Z_{0}=c\right] F_{T_{1}}(t) .
$$

Similarly, we have

$$
\mathbb{E}\left[V_{t} \mid V_{0}=-v\right]=-v \bar{F}_{T_{1}}(t)+\mathbb{E}\left[Z_{1} \mid Z_{0}=-v\right] F_{T_{1}}(t),
$$

and so we find that

$$
\begin{aligned}
\mathbb{E}\left[V_{t}\right]= & \left(c \mathbb{P}\left\{Z_{0}=c\right\}-v \mathbb{P}\left\{Z_{0}=-v\right\}\right) \bar{F}_{T_{1}}(t) \\
& +\left(\mathbb{E}\left[Z_{1} \mid Z_{0}=c\right] \mathbb{P}\left\{Z_{0}=c\right\}+\mathbb{E}\left[Z_{1} \mid Z_{0}=-v\right] \mathbb{P}\left\{Z_{0}=-v\right\}\right) F_{T_{1}}(t) \\
= & \mathbb{E}\left[Z_{0}\right] \bar{F}_{T_{1}}(t)+\mathbb{E}\left[Z_{1}\right] F_{T_{1}}(t) \\
= & \mathbb{E}\left[Z_{0}\right] .
\end{aligned}
$$

The last equality is due to the fact that the random variables $X_{k}$, and so $Z_{k}$, are identically distributed in both the $A=0$ and $A \neq 0$ cases. As a consequence, we obtain

$$
\mathbb{E}\left[S_{t}\right]=t \mathbb{E}\left[Z_{0}\right]
$$

This is the same formula found in [31] for the telegraph process driven by i.i.d. random velocities and an independent homogeneous Poisson process.

In the following sections we discuss some special cases arising in the two different schemes of Bernoulli and Pólya trials, and leading to closed forms for the probability law of $S_{t}$. 


\section{Particular case for the Bernoulli scheme $(A=0)$}

The classical telegraph process is characterized by exponentially distributed times separating consecutive velocity changes. The extension of such a model to the case of velocities driven by Bernoulli trials can be performed in a simple and natural way. Indeed, if we consider a simple telegraph process $\tilde{S}_{t}$ with alternating velocities $c$ and $-v$, and with alternating switching intensities $\tilde{\lambda}:=(1-p) \lambda$ and $\tilde{\mu}:=p \mu$, then it can be proved that the marginal distributions of $\tilde{S}_{t}, t \geq 0$, and $S_{t}, t \geq 0$, are identical (where $S_{t}$ is a telegraph process with switching intensities $\lambda$ and $\mu$ and the same velocities as $\tilde{S}_{t}$, governed by Bernoulli trials with parameter $p$ ). Hence, in this case results for $S_{t}$ can be immediately obtained from those for $\tilde{S}_{t}$. Other results relating to this case, when $\lambda=\mu$, can be found in [31].

With the aim of discussing a nontrivial case, and motivated by previous studies (see [17] and [18]) involving finite-velocity random motions with stochastically decreasing random intertimes, in the following we assume that the random variables $U_{k}$ and $D_{k}$ have exponential distributions with linear rates $\lambda k$ and $\mu k$. Hence, the tail distribution functions are

$$
\bar{F}_{U_{k}}(t)=\mathrm{e}^{-\lambda k t}, \quad \bar{F}_{D_{k}}(t)=\mathrm{e}^{-\mu k t}, \quad t \geq 0,
$$

with $\lambda, \mu>0$. In Figure 2 we present some simulations of $S_{t}$ in the present case, where the particle exhibits a kind of damped motion. This special case belongs to a more general framework in which counting processes with increasing intensity functions are employed in applied fields. A typical example in this respect is the nonhomogeneous Poisson process with increasing intensity function, which deserves interest in reliability contexts involving repairable systems (see, for instance, [9], or [16] for a power-law process). In other cases, as in the present model, the effect of an increasing intensity function can also be obtained by assuming increasing arrival rates (see [8]). Another example of random motion with shrinking steps is the two-dimensional Pearson walk studied in [30]. In [30] the step size decreases deterministically with a geometric rule, whereas in the present model the step length decreases stochastically, according to the tail distribution functions specified in (23).

Due to assumption (23), $U^{(k)}$ and $D^{(k)}, k \geq 1$, have generalized exponential densities

$$
f_{U}^{(k)}(t)=k\left(1-\mathrm{e}^{-\lambda t}\right)^{k-1} \lambda \mathrm{e}^{-\lambda t}, \quad f_{D}^{(k)}(t)=k\left(1-\mathrm{e}^{-\mu t}\right)^{k-1} \mu \mathrm{e}^{-\mu t}, \quad t>0,
$$

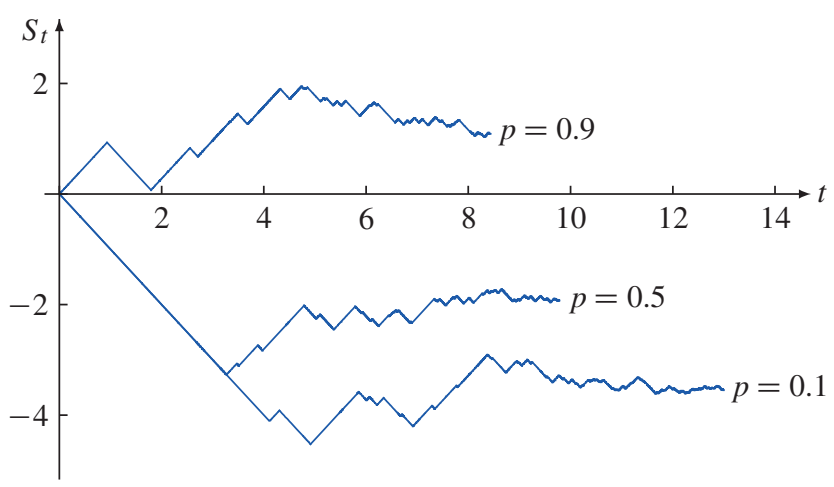

FIGURE 2: Simulated sample paths of $S_{t}$ in the Bernoulli scheme, exponential damped case, with $\lambda=\mu=1$ and $c=v=1$ for various choices of $p$. 
with corresponding cumulative distribution functions

$$
F_{U}^{(k)}(t)=\left(1-\mathrm{e}^{-\lambda t}\right)^{k}, \quad F_{D}^{(k)}(t)=\left(1-\mathrm{e}^{-\mu t}\right)^{k}, \quad t \geq 0 .
$$

Hence, $U^{(k)}$ and $D^{(k)}$ are distributed as the maximum of $k$ i.i.d. random variables having exponential distributions with rates $\lambda$ and $\mu$, respectively.

By making use of the results in the previous section, in the following we obtain the probability law of $S_{t}$. We start by providing the discrete component.

Proposition 3. Let $U_{k}$ and $D_{k}$ be exponentially distributed with rates $\lambda k$ and $\mu k, k=1,2, \ldots$, respectively. For all $t>0$, we have

$$
\mathbb{P}\left\{S_{t}=c t\right\}=\frac{p \mathrm{e}^{-\lambda t}}{1-p\left(1-\mathrm{e}^{-\lambda t}\right)}, \quad \mathbb{P}\left\{S_{t}=-v t\right\}=\frac{(1-p) \mathrm{e}^{-\mu t}}{1-(1-p)\left(1-\mathrm{e}^{-\mu t}\right)} .
$$

Proof. The proof immediately follows from Proposition 1 and (25).

The following theorem and corollary give the absolutely continuous component of the probability law of $S_{t}$.

Theorem 2. Under the assumptions of Proposition 3, for all $t>0$ and $-v t<x<c t$, we have

$$
\begin{aligned}
f(x, t \mid c) & =\frac{(1-p) p \mu \mathrm{e}^{\mu\left(t+\tau_{*}\right)}\left(\mathrm{e}^{\lambda \tau_{*}}-1\right)}{(c+v)\left[p \mathrm{e}^{\mu t}+(1-p) \mathrm{e}^{\left.(\lambda+\mu) \tau_{*}\right]^{2}}\right.}, \\
b(x, t \mid c) & =\frac{(1-p)\left[\lambda p \mathrm{e}^{\lambda \tau_{*}+\mu\left(t+\tau_{*}\right)}+\lambda(1-p) \mathrm{e}^{\lambda \tau_{*}+2 \mu \tau_{*}}\right]}{(c+v)\left[p \mathrm{e}^{\mu t}+(1-p) \mathrm{e}^{(\lambda+\mu) \tau_{*}}\right]^{2}},
\end{aligned}
$$

where $\tau_{*}$ is defined in (17).

Proof. Since $U_{1}$ and $U_{k+1}$ are exponentially distributed with parameters $\lambda$ and $\lambda(k+1)$, respectively, from (12), (15), and (45) below, recalling (23) and (24), we obtain

$$
\begin{aligned}
& f(x, t \mid c) \\
& =\frac{\mu}{c+v} \sum_{k=2}^{+\infty} \sum_{j=0}^{k-2}\left(\begin{array}{c}
k-1 \\
j
\end{array}\right) p^{j+1}(1-p)^{k-1-j}(k-j-1) \mathrm{e}^{-\mu\left(t-\tau_{*}\right)}\left[1-\mathrm{e}^{-\mu\left(t-\tau_{*}\right)}\right]^{k-2-j} \\
& \times \int_{t-\tau_{*}}^{t} \lambda(j+1) \mathrm{e}^{-\lambda\left(s-t+\tau_{*}\right)}\left[1-\mathrm{e}^{-\lambda\left(s-t+\tau_{*}\right)}\right]^{j} \mathrm{e}^{-\lambda(t-s)(j+2)} \mathrm{d} s \\
& =\frac{\mu p(1-p)}{c+v} \mathrm{e}^{-\mu\left(t-\tau_{*}\right)} \sum_{k=2}^{+\infty}(k-1) \sum_{j=0}^{k-2}\left(\begin{array}{c}
k-2 \\
j
\end{array}\right) p^{j}(1-p)^{k-2-j}\left[1-\mathrm{e}^{-\mu\left(t-\tau_{*}\right)}\right]^{k-2-j} \\
& \times \mathrm{e}^{-\lambda \tau_{*}} \int_{t-\tau_{*}}^{t} \lambda(j+1) \mathrm{e}^{-\lambda(t-s)}\left[\mathrm{e}^{-\lambda(t-s)}-\mathrm{e}^{-\lambda \tau_{*}}\right]^{j} \mathrm{~d} s \\
& =\frac{\mu p(1-p)}{c+v} \mathrm{e}^{-\mu\left(t-\tau_{*}\right)-2 \lambda \tau_{*}}\left(\mathrm{e}^{\lambda \tau_{*}}-1\right) \\
& \times \sum_{k=2}^{+\infty}(k-1) \sum_{j=0}^{k-2}\left(\begin{array}{c}
k-2 \\
j
\end{array}\right)\left[p\left(1-\mathrm{e}^{-\lambda \tau_{*}}\right)\right]^{j}\left[(1-p)\left(1-\mathrm{e}^{-\mu\left(t-\tau_{*}\right)}\right)\right]^{k-j-2} \\
& =\frac{\mu p(1-p)}{c+v} \mathrm{e}^{-\mu\left(t-\tau_{*}\right)-2 \lambda \tau_{*}}\left(\mathrm{e}^{\lambda \tau_{*}}-1\right) \frac{\mathrm{e}^{2 \mu t+2 \lambda \tau_{*}}}{\left[(1-p) \mathrm{e}^{(\lambda+\mu) \tau_{*}}+p \mathrm{e}^{\mu t}\right]^{2}} .
\end{aligned}
$$


This yields density (26). Equation (27) can be obtained from (12), (16), and (46) below in a similar way. Indeed, for $k=1$, we have

$$
b_{1}(x, t \mid c)=\frac{\lambda(1-p)}{c+v} \mathrm{e}^{-\lambda \tau_{*}-\mu\left(t-\tau_{*}\right)}
$$

and, for $k \geq 2$,

$$
\begin{aligned}
& b_{k}(x, t \mid c) \\
& =\frac{1}{c+v}\left\{p^{k-1}(1-p) k\left(1-\mathrm{e}^{-\lambda \tau_{*}}\right)^{k-1} \lambda \mathrm{e}^{-\lambda \tau_{*}-\mu\left(t-\tau_{*}\right)}\right. \\
& +\sum_{j=0}^{k-2}\left(\begin{array}{c}
k-1 \\
j
\end{array}\right) p^{j}(1-p)^{k-j}(j+1)\left(1-\mathrm{e}^{-\lambda \tau_{*}}\right)^{j} \lambda \mathrm{e}^{-\lambda \tau_{*}} \\
& \left.\times \int_{\tau_{*}}^{t}(k-j-1)\left(1-\mathrm{e}^{-\mu\left(s-\tau_{*}\right)}\right)^{k-j-2} \mu \mathrm{e}^{-\mu\left(s-\tau_{*}\right)-\mu(k-j)(t-s)} \mathrm{d} s\right\} \\
& =\frac{\lambda}{c+v}\left\{\mathrm{e}^{-\lambda \tau_{*}-\mu\left(t-\tau_{*}\right)}(1-p) k\left[p\left(1-\mathrm{e}^{-\lambda \tau_{*}}\right)\right]^{k-1}\right. \\
& +\mathrm{e}^{-\lambda \tau_{*}} \sum_{j=0}^{k-2}\left(\begin{array}{c}
k-1 \\
j
\end{array}\right)\left[p\left(1-\mathrm{e}^{-\lambda \tau_{*}}\right)\right]^{j}(1-p)^{k-j}(j+1) \\
& \left.\times \mathrm{e}^{-\mu\left(t-\tau_{*}\right)} \int_{\tau_{*}}^{t}(k-j-1)\left[\mathrm{e}^{-\mu(t-s)}-\mathrm{e}^{-\mu\left(t-\tau_{*}\right)}\right]^{k-j-2} \mu \mathrm{e}^{-\mu(t-s)} \mathrm{d} s\right\} \\
& =\frac{\lambda \mathrm{e}^{-\lambda \tau_{*}-\mu\left(t-\tau_{*}\right)}(1-p)}{c+v}\left\{k \alpha^{k-1}+\sum_{j=0}^{k-2}\left(\begin{array}{c}
k-1 \\
j
\end{array}\right)(j+1) \alpha^{j} \beta^{k-1-j}\right\} \text {, }
\end{aligned}
$$

where we have set

$$
\alpha=\alpha(x, t)=p\left(1-\mathrm{e}^{-\lambda \tau_{*}}\right) \quad \text { and } \quad \beta=\beta(x, t)=(1-p)\left(1-\mathrm{e}^{-\mu\left(t-\tau_{*}\right)}\right) .
$$

Hence, recalling that (due to the binomial theorem)

$$
\sum_{j=0}^{k-2}\left(\begin{array}{c}
k-1 \\
j
\end{array}\right)(j+1) \alpha^{j} \beta^{k-1-j}=(\alpha+\beta)^{k-1}+(k-1) \alpha(\alpha+\beta)^{k-2}-k \alpha^{k-1},
$$

from the second equation of (12) we finally obtain

$$
\begin{aligned}
b(x, t \mid c) & =\frac{\lambda \mathrm{e}^{-\lambda \tau_{*}-\mu\left(t-\tau_{*}\right)}(1-p)}{c+v}\left\{1+\sum_{k=2}^{+\infty}(\alpha+\beta)^{k-1}+\alpha \sum_{k=2}^{+\infty}(k-1)(\alpha+\beta)^{k-2}\right\} \\
& =\frac{\lambda \mathrm{e}^{-\lambda \tau_{*}-\mu\left(t-\tau_{*}\right)}(1-p)}{c+v} \frac{1-\beta}{[1-(\alpha+\beta)]^{2}},
\end{aligned}
$$

which coincides with (27).

We are now able to determine the probability density of $S_{t}$ in closed form.

Corollary 1. Under the assumptions of Proposition 3, for all $t>0$ and $-v t<x<c t$, we have

$$
p(x, t \mid c)=\frac{1-p}{c+v} \frac{\lambda(1-p) \mathrm{e}^{(\lambda+2 \mu) \tau_{*}}+p \mathrm{e}^{\mu\left(t+\tau_{*}\right)}\left[(\lambda+\mu) \mathrm{e}^{\lambda \tau_{*}}-\mu\right]}{\left[p \mathrm{e}^{\mu t}+(1-p) \mathrm{e}^{(\lambda+\mu) \tau_{*}}\right]^{2}}
$$


and

$$
p(x, t \mid-v)=\frac{p}{c+v} \frac{\mathrm{e}^{\mu\left(t+\tau_{*}\right)}\left[(\lambda+\mu) \mathrm{e}^{\lambda \tau_{*}}(1-p)+\mu p\right]-\lambda(1-p) \mathrm{e}^{(\lambda+2 \mu) \tau_{*}}}{\left[p \mathrm{e}^{\mu t}+(1-p) \mathrm{e}^{(\lambda+\mu) \tau_{*}}\right]^{2}},
$$

where $\tau_{*}$ is defined in (17). Hence, for $-v t<x<c t$, we have

$$
p(x, t)=\frac{p}{1-p} \frac{1}{s} \frac{\exp \{(\mu-v / s) t-x / s\}}{\left[1+p(1-p)^{-1} \exp \{(\mu-v / s) t-x / s\}\right]^{2}},
$$

where $s:=(c+v) /(\lambda+\mu)$.

Proof. Recalling (11), from densities (26) and (27) we obtain (28). By symmetry, density (29) can be expressed from (28) by replacing $\tau_{*}$ by $t-\tau_{*}$ and interchanging $\lambda$ with $\mu$ and $p$ with $1-p$. Therefore, we finally obtain

$$
p(x, t)=\frac{p(1-p)(\lambda+\mu) \mathrm{e}^{\mu t+(\lambda+\mu) \tau_{*}}}{(c+v)\left[(1-p) \mathrm{e}^{(\lambda+\mu) \tau_{*}}+p \mathrm{e}^{\mu t}\right]^{2}}, \quad-v t<x<c t,
$$

and then (30) easily follows.

Some plots of density (30) are given in Figures 3, 4, and 5 for various choices of the involved parameters.

Remark 4. We can analyse the behavior of density (30) when $x$ tends to the endpoints of the state space $[-v t, c t]$. For $t>0$, we have

$$
\begin{aligned}
\lim _{x \downarrow-v t} p(x, t) & =\frac{p}{1-p} \frac{1}{s} \frac{\mathrm{e}^{\mu t}}{\left[1+p \mathrm{e}^{\mu t} /(1-p)\right]^{2}}, \\
\lim _{x \uparrow c t} p(x, t) & =\frac{p}{1-p} \frac{1}{s} \frac{\mathrm{e}^{-\lambda t}}{\left[1+p \mathrm{e}^{-\lambda t} /(1-p)\right]^{2}} .
\end{aligned}
$$

Straightforward calculations yield the following special case.

Corollary 2. Under the assumptions of Proposition 3, if $\lambda v=\mu c$ then density (30) becomes the truncated logistic density given by

$$
\begin{array}{cl}
p(x, t)=\frac{\mathrm{e}^{-(x-m) / s}}{s\left[1+\mathrm{e}^{-(x-m) / s}\right]^{2}}, & -v t<x<c t, t>0, \\
m=s \ln \left(\frac{p}{1-p}\right), & s=\frac{v}{\mu}=\frac{c}{\lambda} .
\end{array}
$$

Hence, in this case $S_{t}$ admits the stationary density

$$
\lim _{t \rightarrow+\infty} p(x, t)=\frac{\mathrm{e}^{-(x-m) / s}}{s\left[1+\mathrm{e}^{-(x-m) / s}\right]^{2}}, \quad x \in \mathbb{R} .
$$

We note that the right-hand side of (31) is a logistic density with mean $m$ and variance $\pi^{2} s^{2} / 3$. In addition, if $p=\frac{1}{2}$ then the mean $m$ vanishes, and the density identifies with the stationary probability density function of a damped telegraph process, as obtained in Corollary 3.3 of [17]. We note that if $\lambda v \neq \mu c$ then

$$
\lim _{t \rightarrow+\infty} p(x, t)=0, \quad x \in \mathbb{R} .
$$

Finally, let us obtain the mean velocity conditioned by a positive initial velocity. 


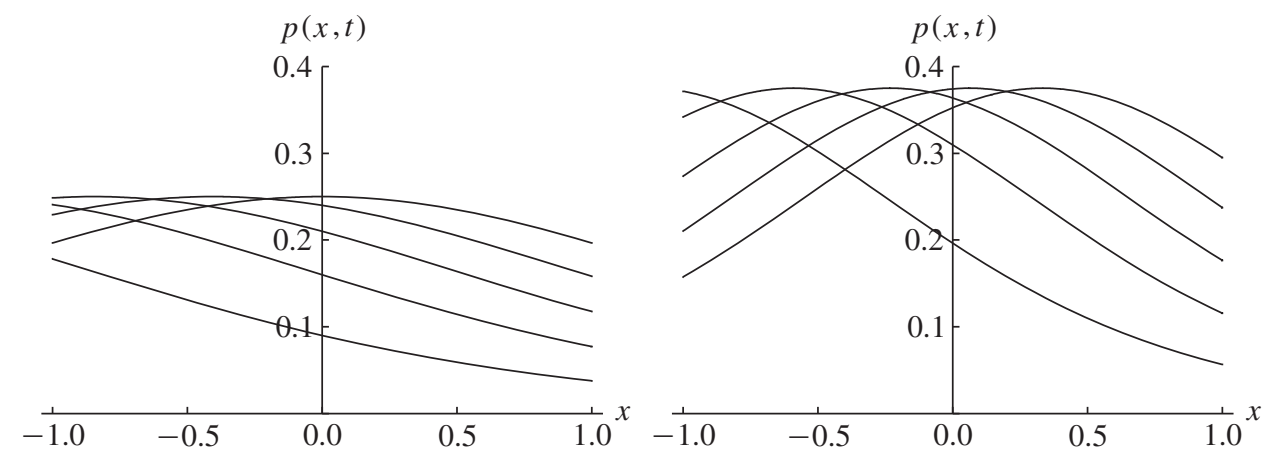

Figure 3: Density (30), with $t=1, c=v=1, \lambda=1$, and $p=0.1,0.2,0.3,0.4,0.5$ (bottom to top near $x=1$ ) for $\mu=1$ (left) and $\mu=2$ (right).
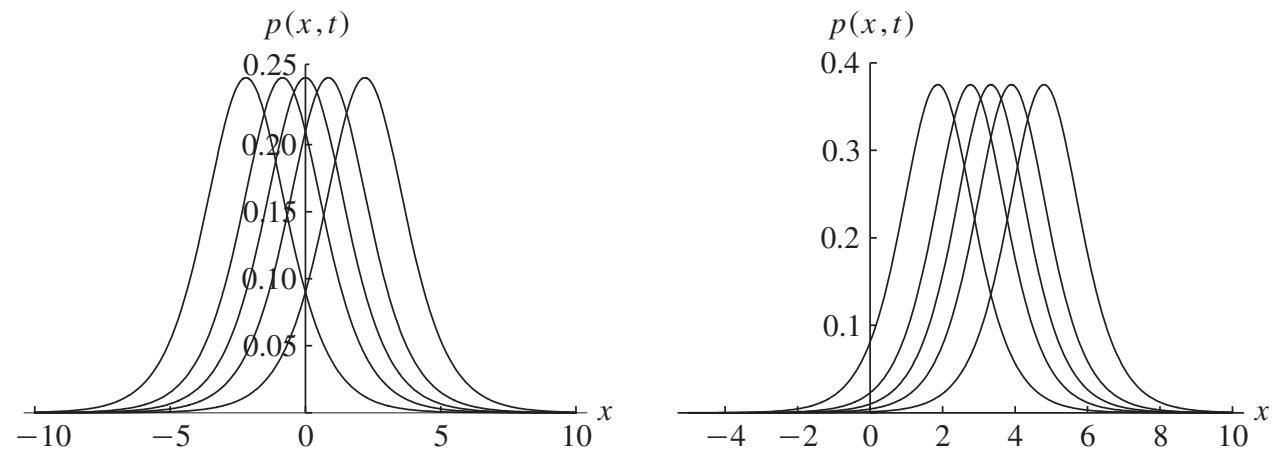

Figure 4: Density (30), with $t=10, c=v=1, \lambda=1$, and $p=0.1,0.3,0.5,0.7,0.9$ (left to right in both plots) for $\mu=1$ (left) and $\mu=2$ (right).

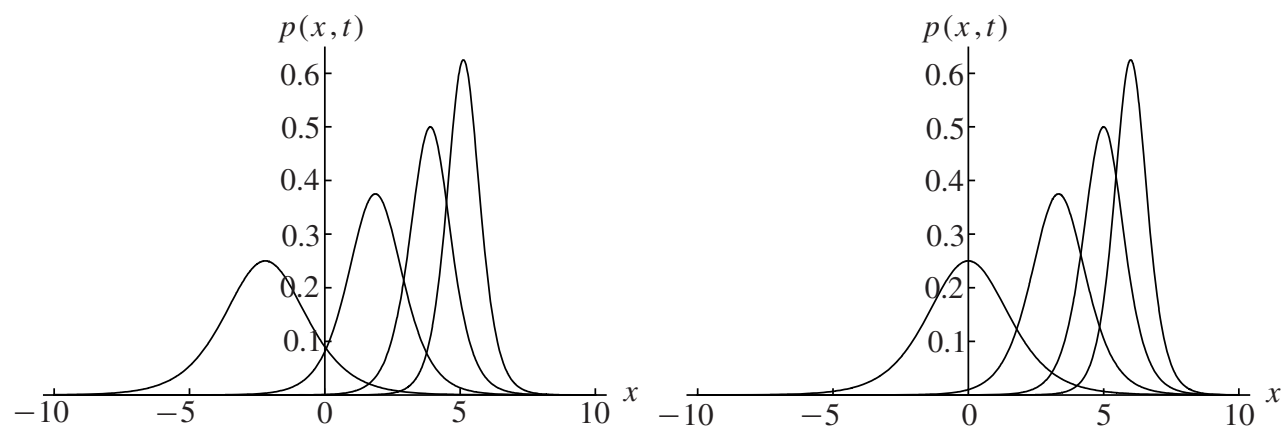

Figure 5: Density (30), with $t=10, c=v=1, \lambda=1$, and $\mu=1,2,3,4$ (left to right in both plots) for $p=0.1$ (left) and $p=0.5$ (right).

Proposition 4. Under the assumptions of Proposition 3, for all $t>0$, we have

$$
\mathbb{E}\left[V_{t} \mid V_{0}=c\right]=c \mathrm{e}^{-\lambda t}+c \pi_{A} \sum_{k=1}^{+\infty} \phi_{k}(t \mid c)+(-v)\left(1-\pi_{A}\right) \sum_{k=1}^{+\infty} \psi_{k}(t \mid c),
$$


where

$$
\begin{aligned}
\phi_{k}(t \mid c)= & \lambda \sum_{j=0}^{k-1}(j+1)\left(\begin{array}{c}
k-1 \\
j
\end{array}\right) p^{j}(1-p)^{k-1-j} \sum_{\ell=0}^{k-j-1} \sum_{h=0}^{j}\left(\begin{array}{c}
k-j-1 \\
\ell
\end{array}\right)\left(\begin{array}{l}
j \\
h
\end{array}\right)(-1)^{\ell+h} \\
\times & {\left[t \mathrm{e}^{-\mu \ell t}{ }_{1} F_{1}(1,2 ;[\mu \ell-\lambda(h+1)] t)\right.} \\
& \left.\quad-\lambda(k+1) \mathrm{e}^{-\lambda(h+1) t} H(\mu \ell-\lambda(h+1), \lambda(k-h) ; t)\right], \\
\psi_{k}(t \mid c)= & \lambda \sum_{j=0}^{k-1}(j+1)\left(\begin{array}{c}
k-1 \\
j
\end{array}\right) p^{j}(1-p)^{k-1-j} \sum_{\ell=0}^{k-j-1} \sum_{h=0}^{j}\left(\begin{array}{c}
k-j-1 \\
\ell
\end{array}\right)\left(\begin{array}{l}
j \\
h
\end{array}\right)(-1)^{\ell+h} \\
\times & {\left[t \mathrm{e}^{-\mu \ell t}{ }_{1} F_{1}(1,2 ;[\mu \ell-\lambda(h+1)] t)\right.} \\
& \left.\quad-\mu(k+1) \mathrm{e}^{-\lambda(h+1) t} H(\mu \ell-\lambda(h+1), \mu(k+1)-\lambda(h+1) ; t)\right],
\end{aligned}
$$

and where the function $H$ is defined by (59) in Appendix B.

Proof. The above formulae easily follow from Theorem 2. Indeed, after some calculations, from (18) and (19), we obtain the cumulative distribution function

$$
\begin{aligned}
F_{T_{k} \mid Z_{0}}(t \mid c)= & \lambda \sum_{j=0}^{k-1}(j+1)\left(\begin{array}{c}
k-1 \\
j
\end{array}\right) p^{j}(1-p)^{k-1-j} \sum_{\ell=0}^{k-j-1} \sum_{h=0}^{j}\left(\begin{array}{c}
k-j-1 \\
\ell
\end{array}\right)\left(\begin{array}{l}
j \\
h
\end{array}\right)(-1)^{\ell+h} \\
& \times t \mathrm{e}^{-\mu \ell t}{ }_{1} F_{1}(1,2 ;[\mu \ell-\lambda(h+1)] t),
\end{aligned}
$$

where ${ }_{1} F_{1}(1,2 ; 0)=1$ and ${ }_{1} F_{1}(1,2 ; z)=\left(\mathrm{e}^{z}-1\right) / z$ for $z \neq 0$. Moreover, the thesis follows by computing the two integrals which appear on the right-hand sides of the equations in (21).

\section{Particular case for the Pólya scheme $(A>0)$}

In this section we consider a special case in which the velocity changes are governed by the Pólya urn scheme. Let us assume that the distributions of $U_{1}$ and $D_{1}$ are $\Gamma(b / A+1, \lambda)$ and $\Gamma(r / A+1, \mu)$, respectively, and that the intertimes $U_{k}$ and $D_{k}$ for $k \geq 2$ are exponential with parameters $\lambda$ and $\mu$, respectively. Therefore, $U^{(k)}$ has gamma distribution $\Gamma(b / A+k, \lambda)$ and $D^{(k)}$ has gamma distribution $\Gamma(r / A+k, \mu)$, so

$$
f_{U}^{(k)}(t)=\frac{\lambda^{b / A+k} t^{b / A+k-1} \mathrm{e}^{-\lambda t}}{\Gamma(b / A+k)}, \quad f_{D}^{(k)}(t)=\frac{\mu^{r / A+k} t^{r / A+k-1} \mathrm{e}^{-\mu t}}{\Gamma(r / A+k)}, \quad t>0 .
$$

Note that $U_{1}$ is stochastically larger than $U_{k}$ and $D_{1}$ is stochastically larger than $D_{k}$ for $k \geq 2$. Hence, the first time interval along both directions is stochastically greater than the other time intervals in the same direction. This is not an unusual assumption, since in renewal theory the distribution of the first interarrival time is often supposed different from that of the other interarrival times (see Chapter 2 of [32] for instance), as for the delayed renewal processes. Moreover, differently from the case treated in the previous section, $U_{1}$ and $D_{1}$ respectively depend on $b, A$ and $r, A$, which are the same parameters involved in the Pólya urn scheme described in Section 2.

Under the above assumptions, we are able to explicitly obtain the probability law of $S_{t}$ in terms of the hypergeometric function ${ }_{1} F_{1}(u, v ; z)$ (see (54) in Appendix B). We first provide 
the tail distribution functions of $U^{(k)}$ and $D^{(k)}$ (see (57) in Appendix B):

$$
\begin{array}{ll}
\bar{F}_{U}^{(k)}(t)=1-\frac{(\lambda t)^{b / A+k} \mathrm{e}^{-\lambda t}}{\Gamma(b / A+k+1)}{ }_{1} F_{1}\left(1, \frac{b}{A}+k+1 ; \lambda t\right), & t \geq 0, \\
\bar{F}_{D}^{(k)}(t)=1-\frac{(\mu t)^{r / A+k} \mathrm{e}^{-\mu t}}{\Gamma(r / A+k+1)}{ }_{1} F_{1}\left(1, \frac{r}{A}+k+1 ; \mu t\right), & t \geq 0 .
\end{array}
$$

In the following proposition we give the discrete component of the probability law of $S_{t}$.

Proposition 5. Let $U_{1}$ and $D_{1}$ be gamma distributed with parameters $(b / A+1, \lambda)$ and $(r / A+$ $1, \mu)$, respectively, and let $U_{k}$ and $D_{k}$ for $k \geq 2$ be exponentially distributed with parameters $\lambda$ and $\mu$, respectively. For all $t>0$, we have

$$
\mathbb{P}\left\{S_{t}=c t\right\}=\frac{b}{b+r} \bar{F}_{U_{1}}(t)+\frac{b(\lambda t)^{b / A} \mathrm{e}^{-\lambda t}}{(b+r) \Gamma((b+A) / A)}\left[{ }_{1} F_{1}\left(1, \frac{b+A+r}{A} ; \lambda t\right)-1\right],
$$

where $\bar{F}_{U_{1}}(t)$ is given by (33) for $k=1$. Similarly,

$$
\mathbb{P}\left\{S_{t}=-v t\right\}=\frac{r}{b+r} \bar{F}_{D_{1}}(t)+\frac{r(\mu t)^{r / A} \mathrm{e}^{-\mu t}}{(b+r) \Gamma((r+A) / A)}\left[{ }_{1} F_{1}\left(1, \frac{b+A+r}{A} ; \mu t\right)-1\right],
$$

where $\bar{F}_{D_{1}}(t)$ is given by (34) for $k=1$.

Proof. Equation (35) follows from (7) using (32). Indeed, since

$$
F_{U}^{(k)}(t)-F_{U}^{(k+1)}(t)=\int_{0}^{t} f_{U}^{(k)}(s) \bar{F}_{U_{k+1}}(t-s) \mathrm{d} s,
$$

we obtain

$$
\begin{aligned}
\mathbb{P}\left\{S_{t}=c t\right\} & =\frac{b}{b+r} \bar{F}_{U_{1}}(t)+\sum_{k=1}^{+\infty} \mathbb{P}\left\{Z_{0}=c, N_{k}=k\right\} \int_{0}^{t} \lambda^{b / A+k} \frac{s^{b / A+k-1} \mathrm{e}^{-\lambda s}}{\Gamma(b / A+k)} \mathrm{e}^{-\lambda(t-s)} \mathrm{d} s \\
& =\frac{b}{b+r} \bar{F}_{U_{1}}(t)+\frac{b \mathrm{e}^{-\lambda t}(\lambda t)^{b / A}}{(b+r) \Gamma((b+A) / A)} \sum_{k=1}^{+\infty} \frac{(1)_{k}}{((b+A+r) / A)_{k}} \frac{(\lambda t)^{k}}{k !} \\
& =\frac{b}{b+r} \bar{F}_{U_{1}}(t)+\frac{b \mathrm{e}^{-\lambda t}(\lambda t)^{b / A}}{(b+r) \Gamma((b+A) / A)}\left[{ }_{1} F_{1}\left(1, \frac{b+A+r}{A} ; \lambda t\right)-1\right] .
\end{aligned}
$$

By interchanging $\lambda$ with $\mu, b$ with $r$, and $U$ with $D$ in (35), we immediately obtain (36).

For the absolutely continuous component of the probability law of $S_{t}$, we have the following results.

Theorem 3. Under the assumptions of Proposition 5, for all $t>0$ and $-v t<x<c t$, we have

$$
f(x, t \mid c)=\frac{\xi\left(\tau_{*}, t\right) \eta\left(\tau_{*}, t\right)}{t-\tau_{*}}
$$

and

$$
\begin{aligned}
b(x, t \mid c)= & \frac{r \lambda\left(\lambda \tau_{*}\right)^{b / A-1} \mathrm{e}^{-\lambda \tau_{*}}}{(c+v) A \Gamma((b+A) / A)} \bar{F}_{D_{1}}\left(t-\tau_{*}\right)\left[{ }_{1} F_{1}\left(1, \frac{b+A+r}{A} ; \lambda \tau_{*}\right)-1\right] \\
& +\frac{\xi\left(\tau_{*}, t\right) \eta\left(\tau_{*}, t\right)}{\tau_{*}}
\end{aligned}
$$


where $\tau_{*}$ is defined in (17), and where

$$
\begin{aligned}
\xi\left(\tau_{*}, t\right):= & \exp \left\{-\lambda \tau_{*}-\mu\left(t-\tau_{*}\right)\right\} \frac{\left(\lambda \tau_{*}\right)^{b / A+1}\left[\mu\left(t-\tau_{*}\right)\right]^{r / A}}{(c+v) \Gamma((b+A) / A) \Gamma(r / A)} \\
\eta\left(\tau_{*}, t\right):= & {\left[\lambda \tau_{*}+\mu\left(t-\tau_{*}\right)\right]^{-1} } \\
& \times\left[{ }_{1} F_{1}\left(1, \frac{b+A+r}{A} ; \lambda \tau_{*}+\mu\left(t-\tau_{*}\right)\right)-1-\frac{A\left[\lambda \tau_{*}+\mu\left(t-\tau_{*}\right)\right]}{b+A+r}\right] \\
& -\left(\lambda \tau_{*}\right)^{-1}\left[{ }_{1} F_{1}\left(1, \frac{b+A+r}{A} ; \lambda \tau_{*}\right)-1-\frac{A \lambda \tau_{*}}{b+A+r}\right]
\end{aligned}
$$

Proof. Owing to (12), (16), and (50) below, and recalling (32), under the given assumptions, we have

$$
\begin{aligned}
f(x, t \mid c)= & \frac{1}{c+v} \sum_{k=2}^{+\infty} \sum_{j=0}^{k-2} \mathbb{P}\left\{N_{k-1}=j, Z_{k}=c \mid Z_{0}=c\right\} \\
& \times \int_{t-\tau_{*}}^{t} \frac{\lambda^{b / A+j+1}\left(s-t+\tau_{*}\right)^{b / A+j}}{\Gamma(b / A+j+1)} \mathrm{e}^{-\lambda\left(s-t+\tau_{*}\right)} \frac{\mu^{r / A+k-j-1}\left(t-\tau_{*}\right)^{r / A+k-j-2}}{\Gamma(r / A+k-j-1)} \\
& \times \mathrm{e}^{-\mu\left(t-\tau_{*}\right)} \mathrm{e}^{-\lambda(t-s)} \mathrm{d} s \\
= & \frac{\xi\left(\tau_{*}, t\right)}{t-\tau_{*}} \sum_{k=2}^{+\infty} \frac{1}{((b+A+r) / A)_{k}} \sum_{j=0}^{k-2}\left(\begin{array}{c}
k-1 \\
j
\end{array}\right)\left(\lambda \tau_{*}\right)^{j}\left[\mu\left(t-\tau_{*}\right)\right]^{k-1-j} \\
= & \frac{\xi\left(\tau_{*}, t\right)}{t-\tau_{*}} \sum_{k=2}^{+\infty} \frac{(1)_{k}}{((b+A+r) / A)_{k}} \frac{1}{k !}\left\{\left[\lambda \tau_{*}+\mu\left(t-\tau_{*}\right)\right]^{k-1}-\left(\lambda \tau_{*}\right)^{k-1}\right\} \\
= & \frac{\xi\left(\tau_{*}, t\right)}{t-\tau_{*}}\left\{[ \lambda \tau _ { * } + \mu ( t - \tau _ { * } ) ] ^ { - 1 } \left[{ }_{1} F_{1}\left(1, \frac{b+A+r}{A} ; \lambda \tau_{*}+\mu\left(t-\tau_{*}\right)\right)-1\right.\right. \\
& \left.-\left(\lambda \tau_{*}\right)^{-1}\left[{ }_{1} F_{1}\left(1, \frac{b+A+r}{A} ; \lambda \tau_{*}\right)-1-\frac{\left.A \lambda \tau_{*}+\mu\left(t-\tau_{*}\right)\right]}{b+A+r}\right]\right\} .
\end{aligned}
$$

Similarly, (12), (16), and (51) below give

$$
\begin{aligned}
& b(x, t \mid c)=\frac{1}{c+v}\left\{\sum_{k=1}^{+\infty} \mathbb{P}\left\{N_{k-1}=k-1, Z_{k}=-v \mid Z_{0}=c\right\}\right. \times \frac{\lambda^{b / A+k} \tau_{*}^{b / A+k-1} \mathrm{e}^{-\lambda \tau_{*}}}{\Gamma(b / A+k)} \bar{F}_{D_{1}}\left(t-\tau_{*}\right) \\
&+\sum_{k=2}^{+\infty} \sum_{j=0}^{k-2} \mathbb{P}\left\{N_{k-1}=j, Z_{k}=-v \mid Z_{0}=c\right\} \\
& \times \frac{\lambda^{b / A+j+1} \tau_{*}^{b / A+j} \mu^{r / A+k-j-1} \mathrm{e}^{-\lambda \tau_{*}-\mu\left(t-\tau_{*}\right)}}{\Gamma(b / A+j+1) \Gamma(r / A+k-j-1)} \\
&\left.\times \int_{\tau_{*}}^{t}\left(s-\tau_{*}\right)^{r / A+k-j-2} \mathrm{~d} s\right\}
\end{aligned}
$$




$$
\begin{aligned}
& =\frac{r\left(\lambda \tau_{*}\right)^{b / A}}{A \Gamma((b+A) / A) \tau_{*}(c+v)} \mathrm{e}^{-\lambda \tau_{*}} \bar{F}_{D_{1}}\left(t-\tau_{*}\right) \sum_{k=1}^{+\infty} \frac{(1)_{k}}{((b+A+r) / A)_{k}} \frac{\left(\lambda \tau_{*}\right)^{k}}{k !} \\
& +\frac{\xi\left(\tau_{*}, t\right)}{\tau_{*}} \sum_{k=2}^{+\infty} \frac{1}{((b+A+r) / A)_{k}} \sum_{j=0}^{k-2}\left(\begin{array}{c}
k-1 \\
j
\end{array}\right)\left(\lambda \tau_{*}\right)^{j}\left[\mu\left(t-\tau_{*}\right)\right]^{k-1-j}
\end{aligned}
$$

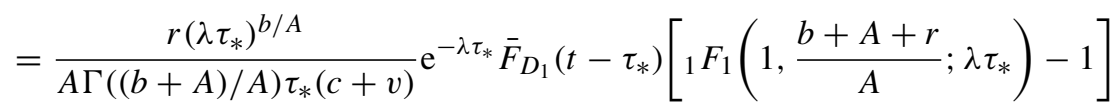

$$
\begin{aligned}
& +\frac{\xi\left(\tau_{*}, t\right)}{\tau_{*}} \sum_{k=2}^{+\infty} \frac{(1)_{k}}{((b+A+r) / A)_{k}} \frac{1}{k !}\left[\left[\lambda \tau_{*}+\mu\left(t-\tau_{*}\right)\right]^{k-1}-\left(\lambda \tau_{*}\right)^{k-1}\right]
\end{aligned}
$$

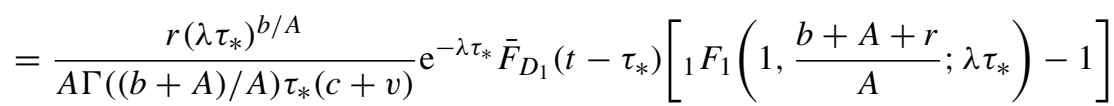

$$
\begin{aligned}
& +\frac{\xi\left(\tau_{*}, t\right)}{\tau_{*}}\left\{[ \lambda \tau _ { * } + \mu ( t - \tau _ { * } ) ] ^ { - 1 } \left[{ }_{1} F_{1}\left(1, \frac{b+A+r}{A} ; \lambda \tau_{*}+\mu\left(t-\tau_{*}\right)\right)-1\right.\right. \\
& \left.-\frac{A\left[\lambda \tau_{*}+\mu\left(t-\tau_{*}\right)\right]}{b+A+r}\right] \\
& \left.-\left(\lambda \tau_{*}\right)^{-1}\left[{ }_{1} F_{1}\left(1, \frac{b+A+r}{A} ; \lambda \tau_{*}\right)-1-\frac{A\left(\lambda \tau_{*}\right)}{b+A+r}\right]\right\} \text {. }
\end{aligned}
$$

This completes the proof.

Corollary 3. Under the assumptions of Theorem 3, for all $t>0$ and $-v t<x<c t$, the probability density of $S_{t}$ is given by (10), where

$$
\begin{aligned}
p(x, t \mid c)= & \frac{r \lambda\left(\lambda \tau_{*}\right)^{b / A-1} \mathrm{e}^{-\lambda \tau_{*}}}{(c+v) A \Gamma((b+A) / A)} \bar{F}_{D_{1}}\left(t-\tau_{*}\right)\left[{ }_{1} F_{1}\left(1, \frac{b+A+r}{A} ; \lambda \tau_{*}\right)-1\right] \\
& +\frac{\xi\left(\tau_{*}, t\right) \eta\left(\tau_{*}, t\right) t}{\tau_{*}\left(t-\tau_{*}\right)}, \\
p(x, t \mid-v)= & \frac{b \mu\left[\mu\left(t-\tau_{*}\right)\right]^{r / A-1} \mathrm{e}^{-\mu\left(t-\tau_{*}\right)}}{(c+v) A \Gamma((r+A) / A)} \bar{F}_{U_{1}}\left(\tau_{*}\right)\left[{ }_{1} F_{1}\left(1, \frac{b+A+r}{A} ; \mu\left(t-\tau_{*}\right)\right)-1\right] \\
& +\frac{\tilde{\xi}\left(\tau_{*}, t\right) \tilde{\eta}\left(\tau_{*}, t\right) t}{\tau_{*}\left(t-\tau_{*}\right)},
\end{aligned}
$$

where $\tau_{*}, \xi$, and $\eta$ are respectively defined in (17), (39), and (40), and where

$$
\begin{aligned}
\tilde{\xi}\left(\tau_{*}, t\right):= & \exp \left\{-\lambda \tau_{*}-\mu\left(t-\tau_{*}\right)\right\} \frac{\left(\lambda \tau_{*}\right)^{b / A}\left[\mu\left(t-\tau_{*}\right)\right]^{r / A+1}}{(c+v) \Gamma((r+A) / A) \Gamma(b / A)}, \\
\tilde{\eta}\left(\tau_{*}, t\right):= & {\left[\lambda \tau_{*}+\mu\left(t-\tau_{*}\right)\right]^{-1} } \\
& \times\left[{ }_{1} F_{1}\left(1, \frac{b+A+r}{A} ; \lambda \tau_{*}+\mu\left(t-\tau_{*}\right)\right)-1-\frac{A\left[\lambda \tau_{*}+\mu\left(t-\tau_{*}\right)\right]}{b+A+r}\right] \\
& -\left[\mu\left(t-\tau_{*}\right)\right]^{-1}\left[{ }_{1} F_{1}\left(1, \frac{b+A+r}{A} ; \mu\left(t-\tau_{*}\right)\right)-1-\frac{A \mu\left(t-\tau_{*}\right)}{b+A+r}\right] .
\end{aligned}
$$

Proof. Equation (41) immediately follows from (11). From Remark 1, by interchanging $\mu$ with $\lambda, t-\tau_{*}$ with $\tau_{*}, b$ with $r$, and $D$ with $U$ in $b(x, t \mid c)$, given in (38), we obtain the 
following density:

$$
\begin{aligned}
f(x, t \mid-v)= & \frac{b \mu\left[\mu\left(t-\tau_{*}\right)\right]^{r / A-1} \mathrm{e}^{-\mu\left(t-\tau_{*}\right)}}{(c+v) A \Gamma((r+A) / A)} \bar{F}_{U_{1}}\left(\tau_{*}\right)\left[{ }_{1} F_{1}\left(1, \frac{b+A+r}{A} ; \mu\left(t-\tau_{*}\right)\right)-1\right] \\
& +\frac{\tilde{\xi}\left(\tau_{*}, t\right) \tilde{\eta}\left(\tau_{*}, t\right)}{t-\tau_{*}} .
\end{aligned}
$$

Similarly, we obtain the density $b(x, t \mid-v)$ by interchanging $\mu$ with $\lambda, t-\tau_{*}$ with $\tau_{*}, b$ with $r$, and $D$ with $U$ in $f(x, t \mid c)$, given in (37), so that

$$
b(x, t \mid-v)=\frac{\tilde{\xi}\left(\tau_{*}, t\right) \tilde{\eta}\left(\tau_{*}, t\right)}{\tau_{*}} .
$$

Hence, (42) now follows from (11).

Various plots of the density $p(x, t)$ given in Corollary 3 are shown by Figures 6, 7, and 8 .

Remark 5. From the formulae given in Theorem 3 we have

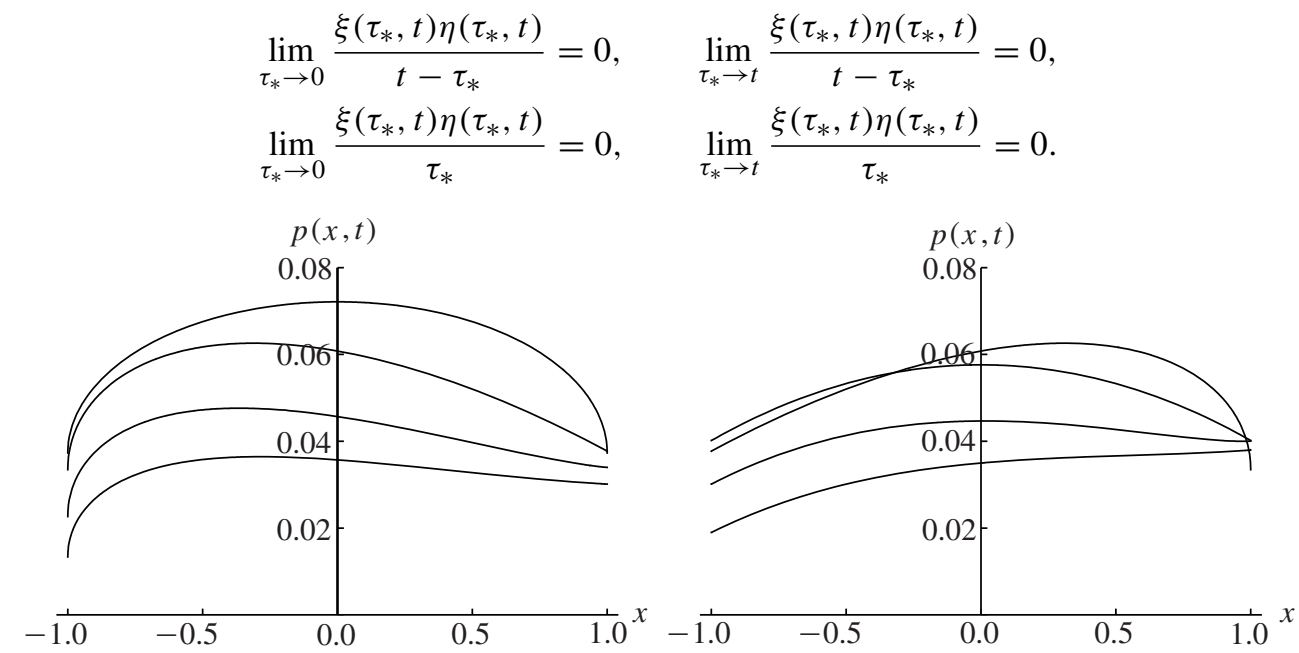

Figure 6: The density $p(x, t)$ for the Pólya scheme with densities (32), $t=1, c=v=1, \lambda=\mu=1$, $A=2$, and $r=1,2,3,4$ (bottom to top near $x=0$ ) for $b=1$ (left) and $b=2$ (right).
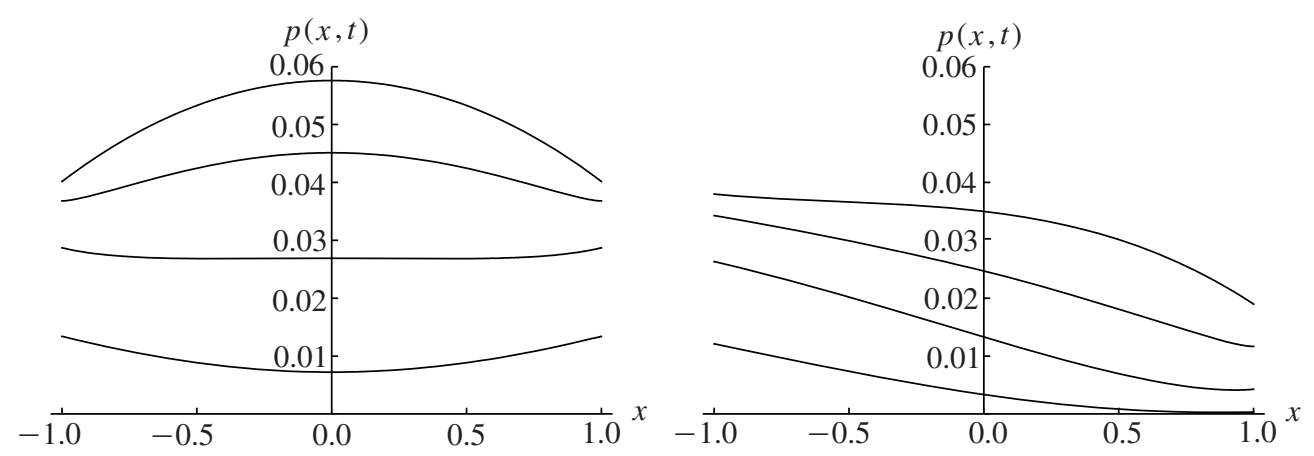

Figure 7: The density $p(x, t)$ for the Pólya scheme with densities (32), $t=1, c=v=1, \lambda=\mu=1$, $r=1$, and $A=0.4,0.6,0.8,1$ (bottom to top near $x=0$ ) for $b=1$ (left) and $b=2$ (right). 

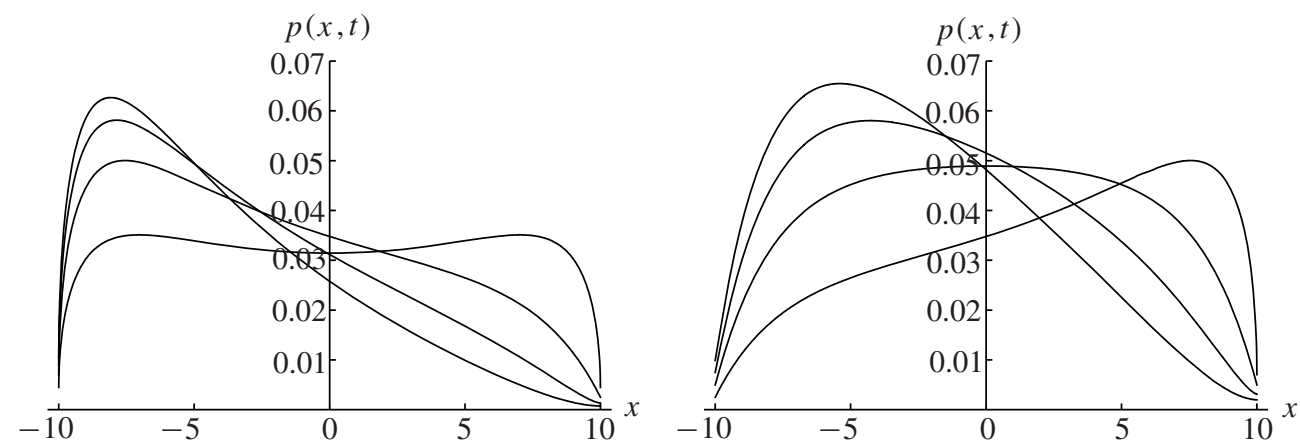

Figure 8: The density $p(x, t)$ for the Pólya scheme with densities (32), $t=10, c=v=1, \lambda=\mu=1$, $A=2$, and $r=1,2,3,4$ (bottom to top near $x=-8$ ) for $b=1$ (left) and $b=2$ (right).

Hence, some calculations allow us to study the behavior of the density $p(x, t)$ in proximity of the endpoints $-v t$ and $c t$. We have

$$
\lim _{x \uparrow-v t} p(x, t)=\frac{b r \mu(\mu t)^{r / A-1} \mathrm{e}^{-\mu t}}{(b+r)(c+v) A \Gamma(r / A+1)}\left[{ }_{1} F_{1}\left(1, \frac{b+A+r}{A} ; \mu t\right)-1\right]
$$

and

$$
\lim _{x \uparrow c t} p(x, t)=\frac{b r \lambda(\lambda t)^{b / A-1} \mathrm{e}^{-\lambda t}}{(b+r)(c+v) A \Gamma(b / A+1)}\left[{ }_{1} F_{1}\left(1, \frac{b+A+r}{A} ; \lambda t\right)-1\right] .
$$

Note that in this case a stationary distribution does not exist. Indeed,

$$
\lim _{t \rightarrow+\infty} \mathbb{P}\left\{S_{t}=c t\right\}=0, \quad \lim _{t \rightarrow+\infty} \mathbb{P}\left\{S_{t}=-v t\right\}=0, \quad \lim _{t \rightarrow+\infty} p(x, t)=0,
$$

since (see Equation 13.1.4 of [1]), as $|z| \rightarrow+\infty$,

$$
{ }_{1} F_{1}(a, b ; z)=\frac{\Gamma(b)}{\Gamma(a)} \mathrm{e}^{z} z^{a-b}\left[1+O\left(|z|^{-1}\right)\right] \quad \text { for } \operatorname{Re}(z)>0 .
$$

Finally, the mean velocity subordinated to the positive initial velocity can be expressed in the following form.

Proposition 6. Under the assumptions of Theorem 3, for all $t>0$, we have

$$
\mathbb{E}\left[V_{t} \mid V_{0}=c\right]=c \bar{F}_{U_{1}}(t)+c \pi_{A} \sum_{k=1}^{+\infty} \phi_{k}(t \mid c)+(-v)\left(1-\pi_{A}\right) \sum_{k=1}^{+\infty} \psi_{k}(t \mid c)
$$

with

$$
\begin{aligned}
\text { with } & \begin{aligned}
\phi_{k}(t \mid c)=\sum_{j=0}^{k-1} \mathbb{P}\left\{N_{k-1}=j \mid Z_{0}=c\right\} & {\left[G\left(\frac{r}{A}+k-j-1, \mu, \frac{b}{A}+j+1, \lambda ; t\right)\right.} \\
& \left.-G\left(\frac{r}{A}+k-j-1, \mu, \frac{b}{A}+j+2, \lambda ; t\right)\right], \\
\psi_{k}(t \mid c)=\sum_{j=0}^{k-1} \mathbb{P}\left\{N_{k-1}=j \mid Z_{0}=c\right\} & {\left[G\left(\frac{b}{A}+j+1, \lambda, \frac{r}{A}+k-j-1, \mu ; t\right)\right.} \\
& \left.-G\left(\frac{b}{A}+j+1, \lambda, \frac{r}{A}+k-j, \mu ; t\right)\right],
\end{aligned}
\end{aligned}
$$

where $\mathbb{P}\left\{N_{k-1}=j \mid Z_{0}=c\right\}$ is given by (49) and the function $G$ is defined by (58) in Appendix $B$. 
Proof. Equations (43) and (44) easily follow from Proposition 2, Remark 2, and (58) since, for $k \geq 1, U_{k+1}$ and $D_{k+1}$ are exponential with parameters $\lambda$ and $\mu$, respectively, and $U^{(k)}$ and $D^{(k)}$ are gamma distributed with parameters $(b / A+k, \lambda)$ and $(r / A+k, \mu)$, respectively.

\subsection{A suitable extension}

In this section we propose a suitable extension of the model based on the Pólya scheme by replacing the classical Pólya urn with the following randomly reinforced urn (cf. [3], [6], [7], and [10]). An urn contains $b>0$ black balls and $r>0$ red balls. At each epoch $n \geq 1$, a ball is drawn and then replaced together with a random number of balls of the same color. Say that $B_{n} \geq 0$ black balls or $R_{n} \geq 0$ red balls are added to the urn according to whether $X_{n}=1$ or $X_{n}=0$, where $X_{n}$ is the indicator function of the event \{black ball at time $n$. We assume that, for each $n \geq 1$,

$$
\left(B_{n}, R_{n}\right) \text { is independent of }\left(X_{1}, B_{1}, R_{1}, X_{2}, \ldots, B_{n-1}, R_{n-1}, X_{n}\right) .
$$

According to this new model, (2) must be replaced by

$$
\begin{gathered}
\mathbb{P}\left\{Z_{n}=c \mid g_{n}\right\}=\mathbb{P}\left\{X_{n+1}=1 \mid g_{n}\right\}=\frac{b+\sum_{k=1}^{n} B_{k} X_{k}}{b+r+\sum_{k=1}^{n}\left(B_{k} X_{k}+R_{k}\left(1-X_{k}\right)\right)}, \\
\mathbb{P}\left\{Z_{n}=-v \mid g_{n}\right\}=\mathbb{P}\left\{X_{n+1}=0 \mid g_{n}\right\}=\frac{r+\sum_{k=1}^{n} R_{k}\left(1-X_{k}\right)}{b+r+\sum_{k=1}^{n}\left(B_{k} X_{k}+R_{k}\left(1-X_{k}\right)\right)},
\end{gathered}
$$

where

$$
g_{0}=\{\varnothing, \Omega\}, \quad g_{n}=\sigma\left(X_{1}, B_{1}, R_{1}, \ldots, X_{n}, B_{n}, R_{n}\right) .
$$

It has been proven that, if $B_{n}=R_{n}$ for each $n$ (cf. [6] and [10]), or, more generally, if $B_{n} \stackrel{\mathrm{D}}{=} R_{n}$ for each $n$ (cf. [7, p. 538]), where $\stackrel{\text { D }}{=}$ denotes equality in distribution, then $\left(X_{n}\right)_{n \geq 1}$ is a sequence of conditionally, identically distributed random variables (which cannot be exchangeable) and so we have, for $n \geq 2$,

$$
\mathbb{P}\left\{X_{n}=1 \mid X_{1}=1\right\}=\mathbb{E}\left[\frac{b+B_{1}}{b+B_{1}+r}\right], \quad \mathbb{P}\left\{X_{n}=0 \mid X_{1}=1\right\}=\mathbb{E}\left[\frac{r}{b+B_{1}+r}\right] .
$$

Using the above equalities, instead of (22), we can obtain a formula for the mean velocity similar to (20). However, in this case the computation of the conditional probability distribution of $N_{k-1}$ needed in (18) is not an easy task, and is likely to be the object of future investigations.

\section{Appendix A. The conditional probability distribution of $N_{k-1}$}

\section{A.1. Bernoulli scheme $(A=0)$}

Using the independence of $\left\{X_{n} ; n \geq 1\right\}$ and setting $p$ as in (3), we have, for $k \geq 1$ and $0 \leq j \leq k-1$,

$$
\mathbb{P}\left\{N_{k-1}=j \mid Z_{0}=c\right\}=\mathbb{P}\left\{\sum_{h=2}^{k} X_{h}=j \mid X_{1}=1\right\}=\left(\begin{array}{c}
k-1 \\
j
\end{array}\right) p^{j}(1-p)^{k-1-j},
$$

and so

$$
\mathbb{P}\left\{N_{k-1}=j, Z_{k}=c \mid Z_{0}=c\right\}=\left(\begin{array}{c}
k-1 \\
j
\end{array}\right) p^{j+1}(1-p)^{k-1-j}
$$


which simply becomes $\mathbb{P}\left\{Z_{1}=c \mid Z_{0}=c\right\}=p$ for $k=1$ and $j=0$. Similarly, we have

$$
\mathbb{P}\left\{N_{k-1}=j, Z_{k}=-v \mid Z_{0}=c\right\}=\left(\begin{array}{c}
k-1 \\
j
\end{array}\right) p^{j}(1-p)^{k-j} .
$$

Conditioning on $Z_{0}=-v$, we obtain

$$
\mathbb{P}\left\{N_{k-1}=k-1-j, Z_{k}=c \mid Z_{0}=-v\right\}=\left(\begin{array}{c}
k-1 \\
j
\end{array}\right) p^{k-j}(1-p)^{j}
$$

and

$$
\mathbb{P}\left\{N_{k-1}=k-1-j, Z_{k}=-v \mid Z_{0}=-v\right\}=\left(\begin{array}{c}
k-1 \\
j
\end{array}\right) p^{k-1-j}(1-p)^{j+1} .
$$

\section{A.2. Pólya scheme $(A>0)$}

Using the exchangeability of $\left\{X_{n} ; n \geq 1\right\}$, we have, for $k \geq 1$ and $0 \leq j \leq k-1$,

$$
\begin{aligned}
\mathbb{P}\left\{N_{k-1}\right. & \left.=j \mid Z_{0}=c\right\} \\
& =\left(\begin{array}{c}
k-1 \\
j
\end{array}\right) \frac{\Gamma((b+A+r) / A)}{\Gamma((b+A) / A) \Gamma(r / A)} \frac{\Gamma(j+(b+A) / A) \Gamma(k-1-j+r / A)}{\Gamma(k-1+(b+A+r) / A)} \\
& =\left(\begin{array}{c}
k-1 \\
j
\end{array}\right)\left(\frac{b+A}{A}\right)_{j}\left(\frac{r}{A}\right)_{k-j-1}\left[\left(\frac{b+A+r}{A}\right)_{k-1}\right]^{-1},
\end{aligned}
$$

where $(\alpha)_{0}=1$ and $(\alpha)_{j}=\alpha(\alpha+1) \cdots(\alpha+j-1)=\Gamma(\alpha+j) / \Gamma(\alpha)$ (the ascending factorial). For $k \geq 2$, the above formula corresponds to the quantity $\mathbb{P}\left\{\sum_{h=2}^{k} X_{h}=j \mid X_{1}=1\right\}$, i.e. the probability of obtaining $j$ black balls in $k-1$ drawings in a Pólya urn scheme starting from $r$ red balls and $b+A$ black balls. Hence, we obtain

$$
\begin{aligned}
\mathbb{P}\left\{N_{k-1}\right. & \left.=j, Z_{k}=c \mid Z_{0}=c\right\} \\
& =\mathbb{P}\left\{X_{k+1}=1 \mid N_{k-1}=j, X_{1}=1\right\} \mathbb{P}\left\{N_{k-1}=j \mid Z_{0}=c\right\} \\
& =\frac{b+A(j+1)}{b+r+A k} \mathbb{P}\left\{N_{k-1}=j \mid Z_{0}=c\right\} \\
& =\left(\begin{array}{c}
k-1 \\
j
\end{array}\right) \frac{\Gamma((b+A+r) / A) \Gamma(j+1+(b+A) / A) \Gamma(k-1-j+r / A)}{\Gamma((b+A) / A) \Gamma(r / A) \Gamma(k+(b+A+r) / A)} \\
& =\left(\begin{array}{c}
k-1 \\
j
\end{array}\right)\left(\frac{b+A}{A}\right)_{j+1}\left(\frac{r}{A}\right)_{k-j-1}\left[\left(\frac{b+A+r}{A}\right)_{k}\right]^{-1},
\end{aligned}
$$

which simply becomes $\mathbb{P}\left\{Z_{1}=c \mid Z_{0}=c\right\}=(b+A) /(b+r+A)$ for $k=1$ and $j=0$. Similarly, we have

$$
\begin{aligned}
& \mathbb{P}\left\{N_{k-1}=j, Z_{k}=-v \mid Z_{0}=c\right\} \\
& =\mathbb{P}\left\{X_{k+1}=0 \mid N_{k-1}=j, X_{1}=1\right\} \mathbb{P}\left\{N_{k-1}=j \mid Z_{0}=c\right\} \\
& =\frac{r+A(k-j-1)}{b+r+A k} \mathbb{P}\left\{N_{k-1}=j \mid Z_{0}=c\right\} \\
& =\left(\begin{array}{c}
k-1 \\
j
\end{array}\right) \frac{\Gamma((b+A+r) / A) \Gamma(j+(b+A) / A) \Gamma(k-j+r / A)}{\Gamma((b+A) / A) \Gamma(r / A) \Gamma(k+(b+A+r) / A)} \\
& =\left(\begin{array}{c}
k-1 \\
j
\end{array}\right)\left(\frac{b+A}{A}\right)_{j}\left(\frac{r}{A}\right)_{k-j}\left[\left(\frac{b+A+r}{A}\right)_{k}\right]^{-1} \text {. }
\end{aligned}
$$


Conditioning on $Z_{0}=-v$, we obtain

$$
\begin{aligned}
& \mathbb{P}\left\{N_{k-1}=k-1-j, Z_{k}=c \mid Z_{0}=-v\right\} \\
& \quad=\left(\begin{array}{c}
k-1 \\
j
\end{array}\right)\left(\frac{r+A}{A}\right)_{j}\left(\frac{b}{A}\right)_{k-j}\left[\left(\frac{b+A+r}{A}\right)_{k}\right]^{-1}
\end{aligned}
$$

and

$$
\begin{aligned}
& \mathbb{P}\left\{N_{k-1}=k-1-j, Z_{k}=-v \mid Z_{0}=-v\right\} \\
& \quad=\left(\begin{array}{c}
k-1 \\
j
\end{array}\right)\left(\frac{r+A}{A}\right)_{j+1}\left(\frac{b}{A}\right)_{k-j-1}\left[\left(\frac{b+A+r}{A}\right)_{k}\right]^{-1} .
\end{aligned}
$$

\section{Appendix B. Auxiliary functions}

For the reader's convenience, we now provide some formulae used in the proofs.

The confluent hypergeometric function (or Kummer's function) used throughout the paper is defined as (see [1] for details)

$$
{ }_{1} F_{1}(u, v ; z)=\sum_{k=0}^{\infty} \frac{(u)_{k}}{(v)_{k}} \frac{z^{k}}{k !} .
$$

We start by recalling the relation

$$
\begin{gathered}
\qquad{ }_{1} F_{1}(a, b ; z)=\mathrm{e}^{z}{ }_{1} F_{1}(b-a, b ;-z) \\
\text { or, equivalently, }{ }_{1} F_{1}(a, b ;-z)=\mathrm{e}^{-z}{ }_{1} F_{1}(b-a, b ; z) .
\end{gathered}
$$

A useful integration formula is Equation 3.383.1 of [21]:

$$
\int_{0}^{t} y^{\nu-1}(t-y)^{\mu-1} \mathrm{e}^{\beta y} \mathrm{~d} y=\frac{\Gamma(\mu) \Gamma(v)}{\Gamma(\mu+v)} t^{\mu+\nu-1}{ }_{1} F_{1}(v, \mu+v ; \beta t)
$$

when $\operatorname{Re}(\mu)>0$ and $\operatorname{Re}(v)>0$.

Let $X$ and $Y$ be independent gamma-distributed random variables with parameters $(\alpha, \mu)$ and $(\beta, \lambda)$, respectively. By (55) and the integration formula (56), we find that

$$
F_{X}(t)=\frac{(\mu t)^{\alpha}}{\Gamma(\alpha+1)} \mathrm{e}^{-\mu t}{ }_{1} F_{1}(1, \alpha+1 ; \mu t), \quad \mu>0, t \geq 0,
$$

and so, for $\alpha, \beta, \mu, \lambda>0$ and $t \geq 0$,

$$
\begin{aligned}
G(\alpha, \mu, \beta, \lambda ; t) & :=\mathbb{P}(X+Y \leq t) \\
& =\int_{0}^{t} F_{X}(t-y) f_{Y}(y) \mathrm{d} y \\
& =\mu^{\alpha} \lambda^{\beta} t^{\alpha+\beta} \mathrm{e}^{-\mu t} \sum_{h=0}^{+\infty} \frac{(\mu t)^{h}}{\Gamma(\alpha+\beta+h+1)}{ }_{1} F_{1}(\beta, \alpha+\beta+h+1 ;(\mu-\lambda) t) .
\end{aligned}
$$

Note that an alternative expression of $G$ was given in [25] as a series of suitable integrals. Analogously to (58), by (56) we can express the function

$$
H(\alpha, \beta ; t):=\int_{0}^{t}(t-y)_{1} F_{1}(1,2 ; \alpha(t-y)) \mathrm{e}^{-\alpha(t-y)} \mathrm{e}^{-\beta y} \mathrm{~d} y, \quad \alpha, \beta \in \mathbb{R}, t \geq 0,
$$


in terms of the hypergeometric function as

$$
H(\alpha, \beta ; t)= \begin{cases}\frac{t}{\alpha}\left[{ }_{1} F_{1}(1,2 ;-\beta t)-\mathrm{e}^{-\alpha t}{ }_{1} F_{1}(1,2 ;(\alpha-\beta) t)\right], & \alpha \neq 0, \beta \in \mathbb{R}, \\ \frac{t}{\beta}\left[\mathrm{e}^{-\beta t}-{ }_{1} F_{1}(1,2 ;-\beta t)\right], & \alpha=0, \beta \neq 0, \\ \frac{t^{2}}{2}, & \alpha=0, \beta=0,\end{cases}
$$

where we recall that ${ }_{1} F_{1}(1,2 ; z)$ reduces to 1 when $z=0$ and to $\left(\mathrm{e}^{z}-1\right) / z$ when $z \neq 0$.

\section{Acknowledgements}

The authors thank an anonymous referee for useful remarks that improved the paper.

This work was partially supported by MIUR (PRIN 2008)—within the projects 'Mathematical models and computation methods for information processing and transmission in neuronal systems subject to stochastic dynamics' and 'Bayesian methods: theoretical advancements and new applications' - and by the CNR PNR project 'CRISIS Lab'.

\section{References}

[1] Abramowitz, M. And Stegun, I. A. (1970). Handbook of Mathematical Functions with Formulas, Graph, and Mathematical Tables. Dover, New York.

[2] Aldous, D. J. (1985). Exchangeability and related topics. In École d'Été de Probabilités de Saint-Flour, XIII1983 (Lecture Notes Math. 1117), Springer, Berlin, pp. 1-198.

[3] Aletti, G., May, C. AND Secchi, P. (2009). A central limit theorem, and related results, for a two-color randomly reinforced urn. Adv. Appl. Prob. 41, 829-844.

[4] Bartlett, M. S. (1957). Some problems associated with random velocity. Publ. Inst. Statist. Univ. Paris. 6, 261-270.

[5] Beghin, L., Nieddu, L. And Orsingher, E. (2001). Probabilistic analysis of the telegrapher's process with drift by means of relativistic transformations. J. Appl. Math. Stoch. Anal. 14, 11-25.

[6] Berti, P., Pratelli, L. And Rigo, P. (2004). Limit theorems for a class of identically distributed random variables. Ann. Prob. 32, 2029-2052.

[7] Berti, P., Crimaldi, I., Pratelli, L. and Rigo, P. (2011). A central limit theorem and its applications to multicolor randomly reinforced urns. J. Appl. Prob. 48, 527-546.

[8] Brown, M. (2011). Detection of changes of multiple Poisson processes monitored at discrete time points where the arrival rates are unknown. Sequent. Anal. 30, 280-296.

[9] Cohen, A. And Sackrowitz, H. B. (1993). Evaluating tests for increasing intensity of a Poisson process. Technometrics 35, 446-448.

[10] Crimaldi, I. (2009). An almost sure conditional convergence result and an application to a generalized Pólya urn. Internat. Math. Forum 4, 1139-1156.

[11] De Gregorio, A. (2010). Stochastic velocity motions and processes with random time. Adv. Appl. Prob. 42, 1028-1056.

[12] De Gregorio, A. (2012). On random flights with non-uniformly distributed directions. J. Statist. Phys. 147, 382-411.

[13] De Gregorio, A. And Orsingher, E. (2012). Flying randomly in $\mathbb{R}^{d}$ with Dirichlet displacements. Stoch. Process. Appl. 122, 676-713.

[14] Di Crescenzo, A. (2001). On random motions with velocities alternating at Erlang-distributed random times. Adv. Appl. Prob. 33, 690-701.

[15] Di Crescenzo, A. And Martinucci, B. (2007). Random motion with gamma-distributed alternating velocities in biological modeling. In Computer Aided Systems Theory, EUROCAST 2007 (Lecture Notes Comput. Sci. 4739), eds R. Moreno-Díaz et al., Springer, Berlin, pp. 163-170.

[16] Di Crescenzo, A. And Martinucci, B. (2009). On a first-passage-time problem for the compound power-law process. Stoch. Models 25, 420-435.

[17] Di Crescenzo, A. And Martinucci, B. (2010). A damped telegraph random process with logistic stationary distribution. J. Appl. Prob. 47, 84-96. 
[18] Di Crescenzo, A., Martinucci, B. And Zacks, S. (2011). On the damped geometric telegrapher's process. In Mathematical and Statistical Methods for Actuarial Sciences and Finance, eds C. Perna and M. Sibillo, Springer, Dordrecht, pp. 175-182.

[19] Foong, S. K. And Kanno, S. (1994). Properties of the telegrapher's random process with or without a trap. Stoch. Process. Appl. 53, 147-173.

[20] Goldstein, S. (1951). On diffusion by discontinuous movements, and on the telegraph equation. Quart. J. Mech. Appl. Math. 4, 129-156.

[21] Gradshteyn, I. S. And Ryzhik, I. M. (2007). Tables of Integrals, Series, and Products, 7th edn. Academic Press, Amsterdam.

[22] Iacus, S. M. (2001). Statistical analysis of the inhomogeneous telegrapher's process. Statist. Prob. Lett. 55, 83-88.

[23] KAC, M. (1974). A stochastic model related to the telegrapher's equation. Rocky Mount. J. Math. 4, 497-509.

[24] Mahmoud, H. M. (2008). Pólya Urn Models. Chapman \& Hall/CRC.

[25] Moschopoulos, P. G. (1985). The distribution of the sum of independent gamma random variables. Ann. Inst. Statist. Math. 37, 541-544.

[26] Orsingher, E. (1990). Probability law, flow function, maximum distribution of wave-governed random motions and their connections with Kirchoff's laws. Stoch. Process. Appl. 34, 49-66.

[27] Orsingher, E. (1995). Motions with reflecting and absorbing barriers driven by the telegraph equation. Random Operators Stoch. Equat. 1, 9-21.

[28] PInsky, M. A. (1991). Lectures on Random Evolutions. World Scientific, River Edge, NJ.

[29] Pólya, G. (1931). Sur quelques points de la théorie des probabilités. Ann. Inst. H. Poincaré 1, 117-161.

[30] Serino, C. A. And Redner, S. (2010). The Pearson walk with shrinking steps in two dimensions. J. Statist. Mech. Theory Exp. 2010, P01006, 12pp.

[31] Stadje, W. and Zacks, S. (2004). Telegraph processes with random velocities. J. Appl. Prob. 41, $665-678$.

[32] Wolff, R. W. (1989). Stochastic Modeling and the Theory of Queues. Prentice Hall, Englewood Cliffs, NJ.

[33] ZACKS, S. (2004). Generalized integrated telegraph processes and the distribution of related stopping times. J. Appl. Prob. 41, 497-507. 